\title{
The Southern Flanking Fields of the 25 Orionis Group
}

\author{
Peregrine M. McGehee ${ }^{1}$
}

\begin{abstract}
The stellar group surrounding the Be (B1Vpe) star 25 Orionis was discovered to be a pre-main-sequence population by the Centro de Investigaciones de Astronomia (CIDA) Orion Variability Survey and subsequent spectroscopy. We analyze Sloan Digital Sky Survey multi-epoch photometry to map the southern extent of the 25 Ori group and to characterize its pre-main-sequence population. We compare this group to the neighboring Orion OB1a and OB1b subassociations and to active star formation sites (NGC 2068/NGC 2071) within the Lynds 1630 dark cloud. We find that the 25 Ori group has a radius of $1.4^{\circ}$, corresponding to 8-11 pc at the distances of Orion OB1a and OB1b. Given that the characteristic sizes of young open clusters are a few pc or less this suggests that 25 Ori is an unbound association rather than an open cluster. Due to its PMS population having a low Classical T Tauri fraction $(\sim 10 \%)$ we conclude that the 25 Ori group is of comparable age to the 11 Myr Orion OB1a subassociation.
\end{abstract}

Subject headings: open clusters and associations: individual (25 Orionis) - stars: formation - stars: late-type - stars: low-mass, brown dwarfs - stars: pre-mainsequence

\section{Introduction}

The pre-main-sequence 25 Orionis group has been recently identified by the Centro de Investigaciones de Astronomia Variability Survey of Orion (CVSO; Briceño et al. 2005a) on the basis of multi-epoch imaging and follow-up spectroscopy. 25 Ori has also been noted by Kharchenko et al. (2005) as a candidate open cluster, ASCC 16, based on analysis of the All-Sky Compiled Catalogue of 2.5 Million Stars (ASCC-2.5; Kharchenko 2001). This group is named after the Be star 25 Orionis, $\left(\alpha_{2000}, \delta_{2000}\right)=\left(05^{h} 24^{m} 44.92^{s},+01^{\circ} 50^{m} 47.2^{s}\right)$ or $(l, b)$ $=(200.96,-18.2)$. Spectroscopically confirmed candidate group members are found within one degree from 25 Orionis (Briceño et al. 2005b).

\footnotetext{
${ }^{1}$ Los Alamos National Laboratory, LANSCE-IC, MS H820, Los Alamos, NM 87545; peregrine@lanl.gov
} 
We use multi-epoch imaging by the Sloan Digital Sky Survey (SDSS) to select candidate pre-main-sequence (PMS) objects based on variability and location in color-magnitude diagrams. The SDSS imaging within the Orion region spans the declination range of $-1.25^{\circ}$ to $+1.25^{\circ}$, thereby covering the southern edge of the 25 Ori group within $0.6^{\circ}$ of the central Be star.

In this paper we examine the PMS populations near 25 Orionis to determine the observational characteristics and spatial extent of this group. In $\S 2$ we describe the SDSS equatorial imaging survey in the Orion region. $§ 3$ revisits the classification scheme of McGehee et al. (2005) for PMS candidates based on multi-band variability. In $\S 4$ we present our results, and in $\S 5$ we discuss our findings, conclusions, and future work.

\section{Observations}

\subsection{Photometry}

The SDSS low Galactic latitude data which includes the Orion equatorial imaging used in this work are presented by Finkbeiner et al. (2004). The SDSS multi-epoch imaging data used in this paper as well as the selection for candidate low-mass pre-main sequence objects are discussed in McGehee et al. (2005). Here we describe the salient features of the SDSS drift-scanned imaging.

The SDSS obtains deep photometry with asinh magnitude (Lupton, Gunn, \& Szalay 1999) limits (defined by $95 \%$ detection repeatability for point sources) of $u=22.0, g=22.2$, $r=22.2, i=21.2$ and $z=20.5$. These five passbands, ugriz, have effective wavelengths of 3540, 4760, 6290, 7690, and $9250 \AA$, respectively. A technical summary of the SDSS is given by York et al. (2000). The SDSS imaging camera and telescope are described by Gunn et al. (1998) and Gunn et al. (2006), respectively. Ivezić et al. (2004) discuss the data management and photometric quality assessment system.

The Early Data Release and the Data Release One are described by Stoughton et al. (2002) and Abazajian et al. (2003). The former includes an extensive discussion of the data outputs and software. Pier et al. (2003) describe the astrometric calibration of the survey and the network of primary photometric standard stars is described by Smith et al. (2002). The photometric system itself is defined by Fukugita et al. (1996), and the system which monitors the site photometricity by Hogg et al. (2001). Abazajian et al. (2003) discuss the differences between the native SDSS $2.5 \mathrm{~m}$ ugriz system and the $u^{\prime} g^{\prime} r^{\prime} i^{\prime} z^{\prime}$ standard star system defined on the USNO $1.0 \mathrm{~m}$ (Smith et al. 2002). The Two-Micron All Sky Survey 2MASS (2MASS; Skrutskie et al. 1997) obtained nearly complete coverage of the sky in 
$J H K_{s}$.

\subsection{Comparison of CIDA and SDSS Survey Coverage}

The SDSS Orion equatorial data consist of a $2.5^{\circ}$ wide stripe centered on the celestial equator. For this work we consider the $37.5 \mathrm{deg}^{2}$ bounded in R.A. by $5^{h}$ to $6^{h}$ that lies completely within the CVSO survey region. While the temporal sampling by the SDSS imaging is much sparser with at most 7 observations per target over a span of nearly 5 years, the SDSS survey is deeper and thus is able to detect low-mass and very-low-mass objects.

There are 197 spectroscopically confirmed Weak-lined T Tauris (WTTS) and Classical T Tauris (CTTS) found by the CVSO survey (Briceño et al. 2005a). Out of the 76 that are located within the SDSS equatorial scans, we find that 10 are identified in the SDSS low-mass star study (McGehee et al. 2005) which selected for M dwarf colored stars on the basis of $(r-i)>0.6$ and $Q_{r i z}>0.35$, where the latter is a reddening-invariant index formed by the SDSS riz magnitudes (see $\S 3.1$ below). These matches are listed in Table 1 . The complete set of WTTS and CTTS candidates identified in the SDSS survey are presented in Table 2. These tables include object classification based on multi-band variability into non-PMS, WTTS, and CTTS as discussed in $\S 3.2$ below. Within $\S 3.3$ we investigate the effectiveness of selection and classification of PMS objects based solely on variability.

The objects detected by the SDSS low-mass star survey are both faint $(V>15.7)$ and red $\left(V-I_{C}>2.2\right)$, as evident in Figure 1. The single faint object that was not selected by McGehee et al. (2005) is CVSO 157, a highly veiled continuum CTTS, whose $r-i$ and $Q_{\text {riz }}$ colors were in the ranges 0.42 to 0.77 and -0.50 to -0.11 , respectively.

\section{Identification of CTTS Candidates}

\subsection{Reddening-Invariant Indices}

The Orion OB1a and OB1b subassociations comprise a range of star formation environments ranging from older, dispersed populations having low line-of-sight interstellar extinction to very young and embedded systems, such as those associated with the Lynds 1630 dark cloud (Lynds 1962). In order to mitigate the need to deredden the photometry for each object we utilize a number of reddening-invariant indices (colors) and magnitudes to aid in the classfication of the stars in these regions

In this work we employ reddening-invariant indices of the form $Q_{x y z}=(x-y)-(y-z) \times$ 
$E(x-y) / E(y-z)$. For passbands defined at wavelengths less than $1 \mu \mathrm{m}, Q_{x y z}$ is dependent upon the assumed ratio of general to selective extinction $\left(R_{V}=A_{V} / E(B-V)\right.$; Cardelli, Clayton, \& Mathis (1989)). Here $x y z$ refer to the specific passbands, e.g. ugrizJHK$K_{S}$, and $E(x-y)$ is the color excess due to reddening of the $x-y$ color. This definition of reddening-invariant colors follows Johnson \& Morgan (1953) in that in our notation their original $Q$ would be written as $Q_{U B V}$. In the $(x-y, y-z)$ color-color diagram the $Q_{x y z}$ axis is perpendicular to the reddening vector. These indices are discussed in full by McGehee et al. (2005).

We use the extinction tables derived by D. Finkbeiner ${ }^{1}$ to define the coefficients used in defining reddening-invariant colors. These tables contain the $A_{X} / E(B-V)$ values for the SDSS ugriz filters for specific values of $R_{V}$ and source spectra. The values we present here are obtained using an $\mathrm{F}$ dwarf source spectrum and $R_{V}=3.1$ which is the standard extinction law found in the diffuse ISM.

In this work we use optical and near-IR indices $Q_{r i z}=(r-i)-0.987(i-z)$ and $Q_{J H K}=$ $(J-H)-1.563(H-K)$. We also employ reddening-invariant magnitudes constructed from linear combinations of observed magnitudes and colors. Here we use a near-IR reddeninginvariant magnitude $J_{J-H}=J-(J-H) A_{J} / E(J-H)=J-2.76(J-H)$. The numerical terms in the $Q_{J H K}$ and $J_{J-H}$ definitions are based on the reddening coefficients of Schlegel, Finkbeiner, \& Davis (1998). The notation follows that of Babusiaux \& Gilmore (2005) who used $K_{s J-K_{s}}$ to determine distances to red clump stars in the inner Galactic bulge. We chose the combination of the $J$ and $H$ bands to minimize the effect of thermal emission from the inner circumstellar disk.

\subsection{Comparison of Variability and Near-IR Disk Indicators}

In McGehee et al. (2005) we identified candidate PMS objects on the basis of $\sigma_{g}>$ 0.05, where the subset flagged as possible CTTS rather than WTTS were selected by $\sigma_{z}>$ 0.05. Here $\sigma_{g}$ and $\sigma_{z}$ are the standard deviations in the $g$ and $z$ bands measured over all observations for a star. Application of these criteria to a comparison field yielded possible contamination fractions of $8.7 \%$ for all PMS candidates (WTTS and CTTS) and of $2.9 \%$ for CTTS only. The majority of the contaminants are likely foreground active M dwarfs (dMe).

In this work we improve these selection criteria by comparing the SDSS multi-band variability against the intrinsic near-IR excess computed from 2MASS observations. This

\footnotetext{
${ }^{1}$ private communication; see http://www.astro.princeton.edu/ dfink/sdssfilters/
} 
procedure assumes that the high-amplitude variability and near-IR excess are both due to the presence of an inner circumstellar disk with the former generated by magnetospheric accretion processes and the latter by disk thermal emission.

Due to the low-mass and very-low-mass nature of our sample we need to be concerned about the contrast between the stellar photospheric emission and that from the inner circumstellar disk. Liu, Najita, \& Tokunaga (2003) note that the $K$ band excess vanishes for spectral types of M6 and later, thereby making use of longer wavelength bands such as $L$ and $M$ critical for detection of circumstellar disks around very-low-mass stars and young brown dwarfs. In Figure 2 we see that, based on the Baraffe et al. (1998) [BCAH98] isochrones and the Luhman (2003) PMS temperature scale, a 2 Myr old star in the Orion OB1b subassociation with a spectral type between M5 and M6 would have $J_{J-H}=12.0$. We adopt this as the faint magnitude limit for reliable detection of $K$ band disk emission in our study.

We begin by defining a reddening-invariant near-IR excess, $\Delta Q_{J H K}$, relative to the stellar locus in the $\left(Q_{J H K}, J_{J-H}\right)$ color-magnitude diagram. In Figure 3 we present the BCAH98 isochrones for Orion OB1a and Orion OB1b in this reddening-invariant near-IR color-magnitude diagram. The steepening of the stellar locus for the brighter stars motivates us to define a bright limit of $J_{J-H}=9.6$. The isochrones for Orion OB1a and OB1b lie nearly on top of each other for $9.6<J_{J-H}<12.0$. A least-squares fit gives $Q_{J H K}=1.986-$ $0.157 J_{J-H}$ in this magnitude range, thus we define $\Delta Q_{J H K}=Q_{J H K}-\left(1.986-0.157 J_{J-H}\right)$.

In order to determine the reddening-invariant colors of disk candidates we first consider the CTTS locus of Meyer, Calvet, \& Hillenbrand (1997) which, in the $(J-H, H-K)$ colorcolor diagram, is $(J-H)=0.58(H-K)+0.52$. For a star on the CTTS locus an increase in $(H-K)$ of $\delta(H-K)$ implies $\delta Q_{J H K}=-0.98 \delta(H-K)$ and $\delta J_{J-H}=0.58 \delta(H-K)$ with the resulting $\delta \Delta Q_{J H K}=-1.23 \delta(H-K)$. Therefore we expect CTTS candidates to have $\Delta Q_{J H K}<0$, that is, found to the left of the stellar locus in the $\left(Q_{J H K}, J_{J-H}\right)$ diagram.

Examination of Figures 4 and 5, in which the standard deviations in $g$ and $z$ are compared against $\Delta Q_{J H K}$ for stars having $9.6<J_{J-H}<12.0$, reveals that the criteria used by McGehee et al. (2005) for detection of CTTS candidates are insufficently stringent. Specifically, the threshold for variability in the $g$ band that matches the onset of significant near-IR excess is $\sigma_{g}=0.2$, i.e., a factor of four greater than used in McGehee et al. (2005). The stars selected by these new criteria (diamonds) and having $J_{J-H}<12$ show near-IR excess in the $\left(Q_{J H K}, J_{J-H}\right)$ CMD (Figure 3$)$. 


\subsection{The Effectiveness of Classification by Variability Alone}

We see in Table 1 that some discrepancies exist between the results of the variabilitybased classification scheme in this work and those obtained by analysis of optical spectra in Briceño et al. (2005b). Out of the 7 WTTS we find that the SDSS survey agrees on 4 objects, classing the remaining 3 as non-PMS. For the 3 confirmed CTTS, we class 2 as WTTS and 1 as non-PMS.

We use the full sample of Briceño et al. (2005b) to assess the effectiveness of distinguishing WTTS and CTTS based on a threshold value of $\sigma_{V}$. For each threshold value of $\sigma_{V}$ we identify WTTS candidates as those with $\sigma_{V}$ below this threshold and CTTS candidates those RMS values exceeed this threshold. In this collection of stars from both the Orion OB1a and OB1b subassociations there are $N_{W T T S}=138$ WTTS and $N_{C T T S}=38$ CTTS, giving a CTTS or inner disk fraction of $21.7 \%$. We begin by examining the Cumulative Distribution Function (CDF) for $\sigma_{V}$ for both types of PMS stars. In Figure 6 we see that while the CTTS clearly tend to have higher $\sigma_{V}$ values than the WTTS, there do exist both high-amplitude WTTS and low-amplitude CTTS, thus using the amplitude or, in our case, the RMS of the variability will not be perfectly efficient.

To measure the selection efficiency we consider two selection metrics, the accuracy and

the computed CTTS fraction. In order to assess the ability to classify individual stars as WTTS or CTTS we use the accuracy, defined as

$$
\text { Accuracy }=\frac{N_{T P}}{N_{C T T S}}-\frac{N_{F P}}{N_{W T T S}}
$$

where $N_{T P}$ and $N_{F P}$ are the number of true positives and false positives, respectively. We also look at computed CTTS fraction, given by

$$
f_{C T T S}=\frac{N_{T P}+N_{F P}}{N_{C T T S}+N_{W T T S}} .
$$

In Figure 7 we plot both of these selection metrics against the threshold value of $\sigma_{V}$. We find that the peak accuracy of $\sim 0.4$ is found for a threshold value of 0.1 magnitudes and that the computed CTTS fraction is close to the actual CTTS fraction for $\sigma_{V}$ thresholds between 0.1 and 0.15 magnitudes. Since the variability amplitude in CTTS increases sharply at shorter wavelengths, with typical values of $\sigma_{B} / \sigma_{V} \sim 1.3$ (Herbst et al. 1994), we might expect that the optimal threshold for WTTS/CTTS differentiation using the SDSS $g$ band, which has a similar effective wavelength as $B$, may be in the range of 0.13 to 0.2 magnitudes. This suggests that the computed CTTS fraction in our work, which is based on a $\sigma_{g}$ threshold of 0.2 magnitudes, may not be unreasonable. 


\section{Results}

\subsection{Distribution}

The 25 Orionis group is evident in a surface density plot of candidate PMS stars that are identified on the basis of $\sigma_{g}>0.05$. In Figure 8 there are three obvious groupings of PMS stars which, in order of increasing R.A., are the 25 Ori group, the Orion OB1b subassociation, and the NGC 2068/NGC 2071 star formation site in the L1630 cloud. The surface densities are computed on a $1.0^{\circ}$ by $0.25^{\circ}$ grid in R.A. and Dec. with the peak value of 68 stars $\mathrm{deg}^{-2}$ found at the center of the Ori OB1b subassociation.

We look for the southern extent of the 25 Ori group by computing the surface density of the PMS candidates as a function of radial distance from the Be star 25 Orionis. Due to the center of the group being outside of the SDSS imaging area we compute the areas for

each radial bin as $\int_{r}^{r+\Delta r} 2 r \cos ^{-1}(d / r) d r$ where the radial bin size $\Delta r=0.4^{\circ}$, the distance between 25 Ori and the SDSS imaging area $d=0.6^{\circ}$, and $r \geq d$.

In Figure 9 we see that the 25 Ori group blends into the surrounding dispersed PMS population of Ori OB1a at distances greater than $1.4^{\circ}$ from the Be star 24 Orionis. In contrast, the NGC 2068/NGC 2071 complex is much more compact and contained within a radius of $0.6^{\circ}$, as measured from the center of NGC 2071. The near-IR survey of (Lada et al. 1991) of L1630, complete to $K=13$, showed that the four active star formation sites in L1630 are tightly clustered with typical effective radii of $0.1^{\circ}$ or less. Therefore most of this spatial extent is accounted for by the $0.3^{\circ}$ separation between individual NGC 2068 and NGC 2071 protoclusters. Futhermore, the submillimeter dust continuum study of NGC 2068/NGC 2071 at $450 \mu \mathrm{m}$ and $850 \mu \mathrm{m}$ by Motte et al. (2001) shows that each of these protoclusters consist of filamentary dense cores.

In Figure 10, which plots the individual stars within a $4^{\circ}$ by $4^{\circ}$ region centered on 25 Orionis, the clustering of candidate PMS objects around the central Be star is evident. For comparison, Figure 11 shows the same sized region around the NGC 2068/NGC 2071 star formation site. In each of these figures we identify the SDSS low-mass WTTS and CTTS candidates and the spectroscopically confirmed T Tauris from the CVSO.

\subsection{CTTS Fraction}

We examine the relative fraction of CTTS candidates, defined as N(CTTS)/(N(CTTS) $+\mathrm{N}($ WTTS $)$ ), using the same $1.0^{\circ}$ by $0.25^{\circ}$ spatial grid as used by the surface density map (Figure 8). We compute this fraction for each spatial bin that contains five or more PMS 
candidates. As seen in Figure 12 the peak fraction is found in the NGC 2068/NGC 2071 complex, which is an active star formation site. The CTTS fraction is somewhat less in the $\sim 2$ Myr Ori OB1b association, and diminished in the 25 Orionis group.

Examination of the CTTS fraction, using the radial bins defined in Figure 9, shows that the core of the L1630 site has a CTTS fraction of $0.7 \pm 0.35$. This is consistent with the disk fraction of $0.85 \pm 0.15$ found by Haisch et al. (2001) for another L1630 star formation site, the 0.3 Myr NGC 2024 protocluster. In the southern flanking fields of 25 Ori, however, the

CTTS fraction is $\sim 0.1$. This is suggestive of an relatively older population than in L1630 or Orion OB1b since the CTTS fraction decreases with age, due to typical circumstellar disk lifetimes of order 1 to 10 Myr (Kenyon \& Hartmann 1995).

\section{Discussion and Conclusions}

We have mapped the southern extent of the 25 Ori group using multi-epoch imaging by the Sloan Digital Sky Survey. By studying the surface densities of PMS candidates in radial bins centered on the Be star 25 Ori, we see that the low-mass population of the 25 Ori group extends out to $\sim 1.4^{\circ}$. This corresponds to a physical radius of 8 to $11 \mathrm{pc}$, based on distances of 330 pc (for Ori OB1a) and 440 pc (for OB1b), respectively.

The majority of young $(<15$ Myr) clusters have radii less than a few pc (van den Bergh 2005). While there exist young clusters that have significantly larger physical dimensions these are probably not gravitationally bound. The large spatial extent of the 25 Ori group suggests that it is an unbound association, rather than a open cluster, although this is a preliminary classification based only on the southern flanking fields.

Comparison of variability and near-IR excess have led to the adoption of selection criteria for CTTS candidates based on multi-band variability that are $\sigma_{g}>0.2$ and $\sigma_{z}>0.05$, with the $g$ band criterion a factor of four times more restrictive than used in McGehee et al. (2005). By classifying objects on the basis of multi-band variability as probable WTTS or CTTS candidates we detect the known star formation complexes in the Orion equatorial region (L1630 and Orion OB1b) by virtue of their high CTTS fraction, with the core of the NGC 2068/NGC 2071 complex in the northern portion of the L1630 cloud having a CTTS fraction $\sim 0.7$

We find that both the 25 Ori group and the Orion OB1a subassociation have CTTS fractions of $\sim 0.1$, similar to that found by Briceño et al. (2005b). In Figure 12. of Briceño et al. (2005b) we that this CTTS, or inner disk, fraction, is consistent with ages between 5 Myr and 30 Myr. 
Future work involving this group includes mapping of the central region and detailed comparison of its structure with a number of young clusters and associations. Our goal is to determine if a gravitationally bound core to the 25 Ori group exists that may evolve into an open cluster.

We thank the anonymous referee for comments that have improved this paper. P.M.M. acknowledges support from LANL Laboratory Directed Research and Development (LDRD) program 20060495ER. Funding for the SDSS and SDSS-II has been provided by the Alfred P. Sloan Foundation, the Participating Institutions, the National Science Foundation, the U.S. Department of Energy, the National Aeronautics and Space Administration, the Japanese Monbukagakusho, the Max Planck Society, and the Higher Education Funding Council for England. The SDSS Web Site is http://www.sdss.org/.

The SDSS is managed by the Astrophysical Research Consortium for the Participating Institutions. The Participating Institutions are the American Museum of Natural History, Astrophysical Institute Potsdam, University of Basel, Cambridge University, Case Western Reserve University, University of Chicago, Drexel University, Fermilab, the Institute for Advanced Study, the Japan Participation Group, Johns Hopkins University, the Joint Institute for Nuclear Astrophysics, the Kavli Institute for Particle Astrophysics and Cosmology, the Korean Scientist Group, the Chinese Academy of Sciences (LAMOST), Los Alamos National Laboratory, the Max-Planck-Institute for Astronomy (MPA), the Max-Planck-Institute for Astrophysics (MPIA), New Mexico State University, Ohio State University, University of Pittsburgh, University of Portsmouth, Princeton University, the United States Naval Observatory, and the University of Washington.

This publication makes use of data products of the Two Micron All Sky Survey, which is a joint project of the University of Massachusetts and the Infrared Processing and Analysis Center/California Institute of Technology, funded by the National Aeronautics and Space Administration and the National Science Foundation.

Facilities: SDSS, 2MASS

\section{REFERENCES}

Abazajian, K., et al. 2003, AJ, 126, 2081 AJ, 129, 1755

Babusiaux, G. \& Gilmore, G. 2005, in "Resolved Stellar Populations", ASP Conf. Series., eds. D. Valls-Gabaud \& M. Chavez (astro-ph/0506413). 
Baraffe, I. et al. 1998, A\&A, 337, 403 (BCAH98)

Briceño, C. et al. 2005, AJ, 129, 907

Briceño, C. et al. 2005, Star Formation in the Era of the Three Great Observatories, July 13-15, Cambridge, MA.

Cardelli, J. A., Clayton, G .C. \& Mathis. J. S. 1989, ApJ, 345, 245

Finkbeiner, D. et al. 2004, AJ, 128, 2577

Fukugita, M. et al. 1996, AJ, 111, 1748

Gunn, J. E. et al. 1998, AJ, 116, 3040

Gunn, J. E. et al. 2006, AJ, submitted, astro-ph/0602326.

Haisch, K E. et al. 2001, AJ, 121, 1512

Herbst, W. et al. 1994, AJ, 108, 1906

Hogg, D.W., Finkbeiner, D.P., Schlegel, D.J., \& Gunn, J.E. 2001, AJ, 122, 2129

Ivezić, Ž, Lupton, R.H., Schlegel, D. et al. 2004, AN, 325, No. 6-8, 583-9.

Johnson, H.L. \& Morgan, W.W. 1953, ApJ, 117, 313

Kenyon, S. J. \& Hartmann, L. 1995, ApJS, 101, 117

Lada, E.A. et al., ApJ, 371, 171

Liu, M., Najita, J, \& Tokunaga, A. 2003, ApJ, 585, 372

Luhman, K.L. et al. 2003, ApJ, 593, 1093

Lupton, R.H., Gunn, J.E., \& Szalay, A.S. 1999, AJ, 118, 1406

Lynds, B. T. 1962, ApJS, 7, 1

Kharchenko, N.V. 2001, Kinematics and Physics of Celestial Bodies, 17, 409

Kharchenko, N.V., Piskunov, A.E., Roeser, S., Schilbach, E., Scholz, R.-D. 2005, A\&A, 440, 403

McGehee, P.M., West, A.A., Smith, J.A., Anderson, K.S.J., Brinkmann, J. 2005, AJ, 130, 1752 
Meyer, M. R., Calvet, N., \& Hillenbrand, L. A. 1997, AJ, 114, 288

Motte, F. et al. 2001, A\&A, 372, L41

Pier, J.R., Munn, J.A., Hindsley, R.B., Hennessy, G.S., Kent, S.M., Lupton, R.H., \& Ivezić, Ž. 2003, AJ, 125, 1559

Schlegel, D. J., Finkbeiner, D. P., \& Davis, M. 1998, ApJ, 500, 525

Skrutskie, M. F, et al., 1997, The Impact of Large-Scale Near-IR Sky Surveys, F. Garzon et al., eds (Dordrecht:Kluwer)

Smith, J.A., et al. 2002, AJ, 123, 2121

Stoughton, C., et al. 2002, AJ, 123, 485

van den Bergh, S. 2005, astro-ph/0511702

York, D. G. et al. 2000, AJ, 120, 1579 


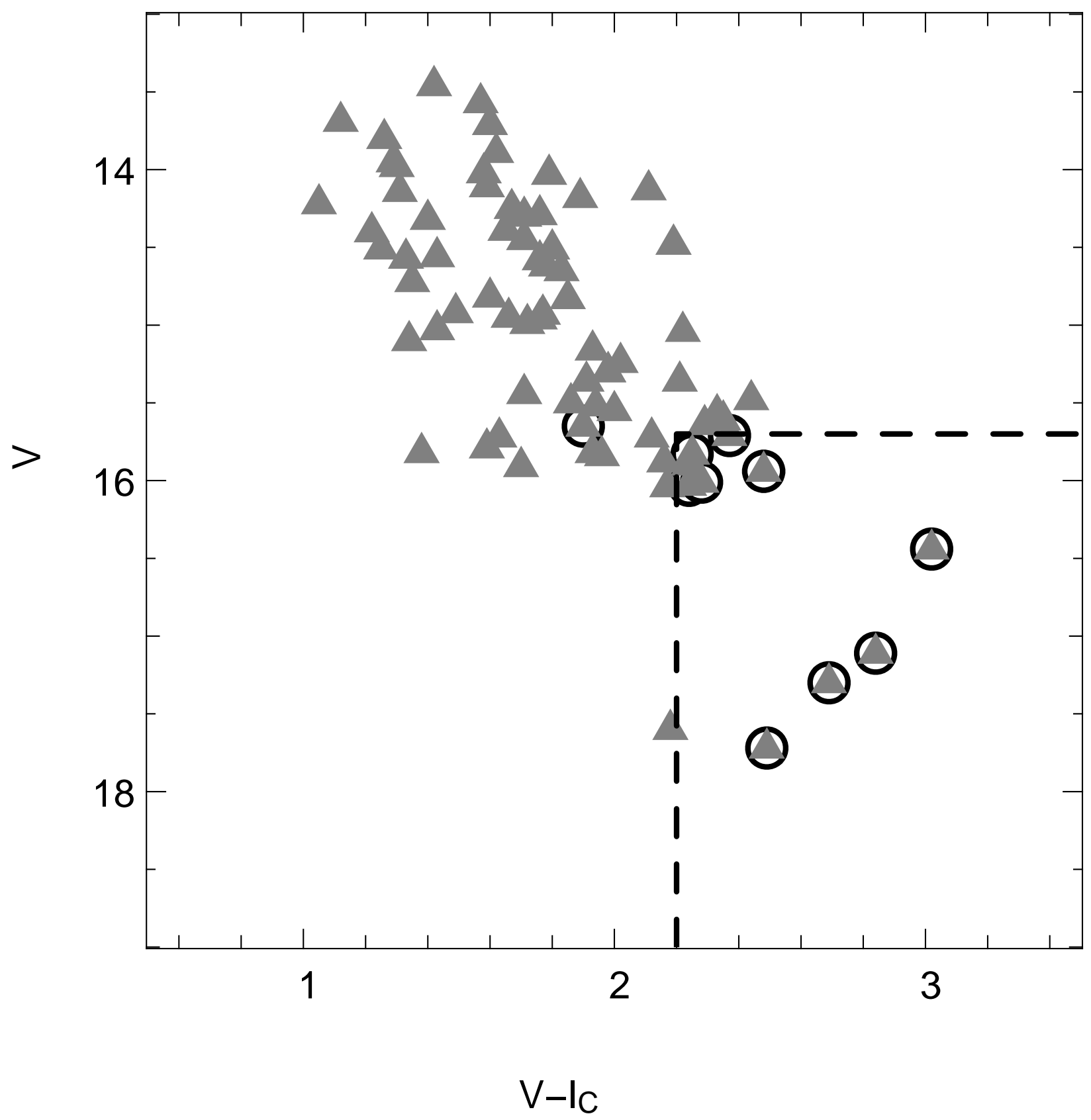

Fig. 1.- $\left(V-I_{c}, V\right)$ color-magnitude diagram for equatorial CIDA objects. The colors and magnitudes of the 76 spectroscopically confirmed pre-main-sequence objects found in the CIDA Orion Variability Survey that lie within the SDSS equatorial scans are shown here. Those objects selected by the SDSS-based low-mass star study of McGehee et al. (2005) are highlighted by open circles. The effective selection region of the SDSS study is bounded by $V-I_{C}>2.2$ and $V>15.7$ (dashed lines). The faint star at $V-I_{C}=2.18, V=17.60$ is the continuum CTTS CVSO 157. 


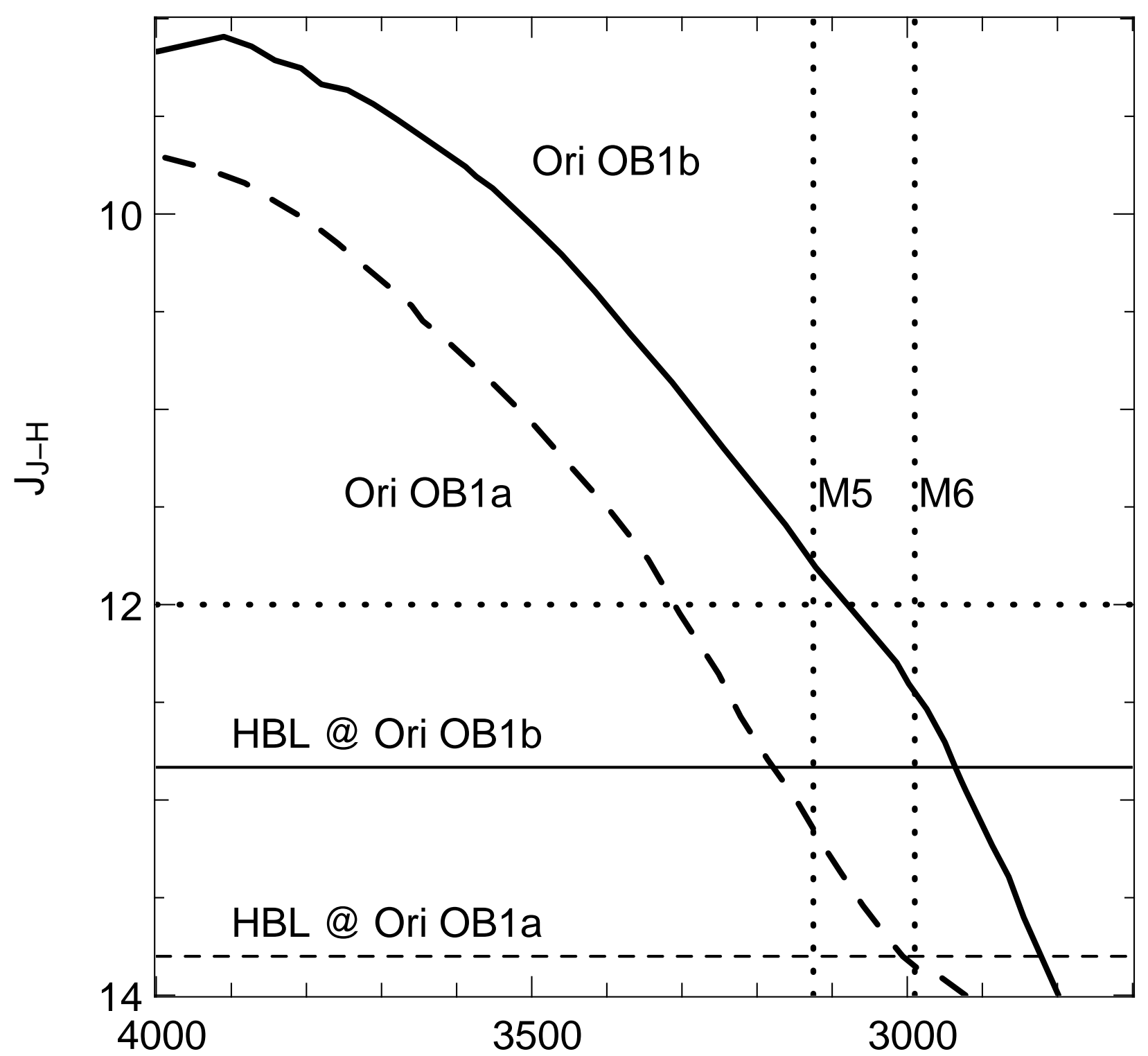

$T_{\text {eff }}$

Fig. 2.- $\left(J_{J-H}, T_{e f f}\right)$ diagram for Orion OB1a and OB1b. The relation between $T_{\text {eff }}$ and $J_{J-H}$ for the Orion OB1a (dashed line) and Orion OB1a (solid line) subassociations are shown here based on the BCAH98 models. The two vertical dotted lines mark spectral types of M5 and M6, assuming the PMS temperature scale of Luhman (2003). The faint magnitude limit of $J_{J-H}=12.0$ for detection of circumstellar disk signatures, based on a lack of contrast in the NIR for spectral types of M6 or later, is traced by the horizontal dotted line. The Hydrogen Burning Limit $\left(0.075 M_{\odot}\right)$ is indicated for both subassociations. 


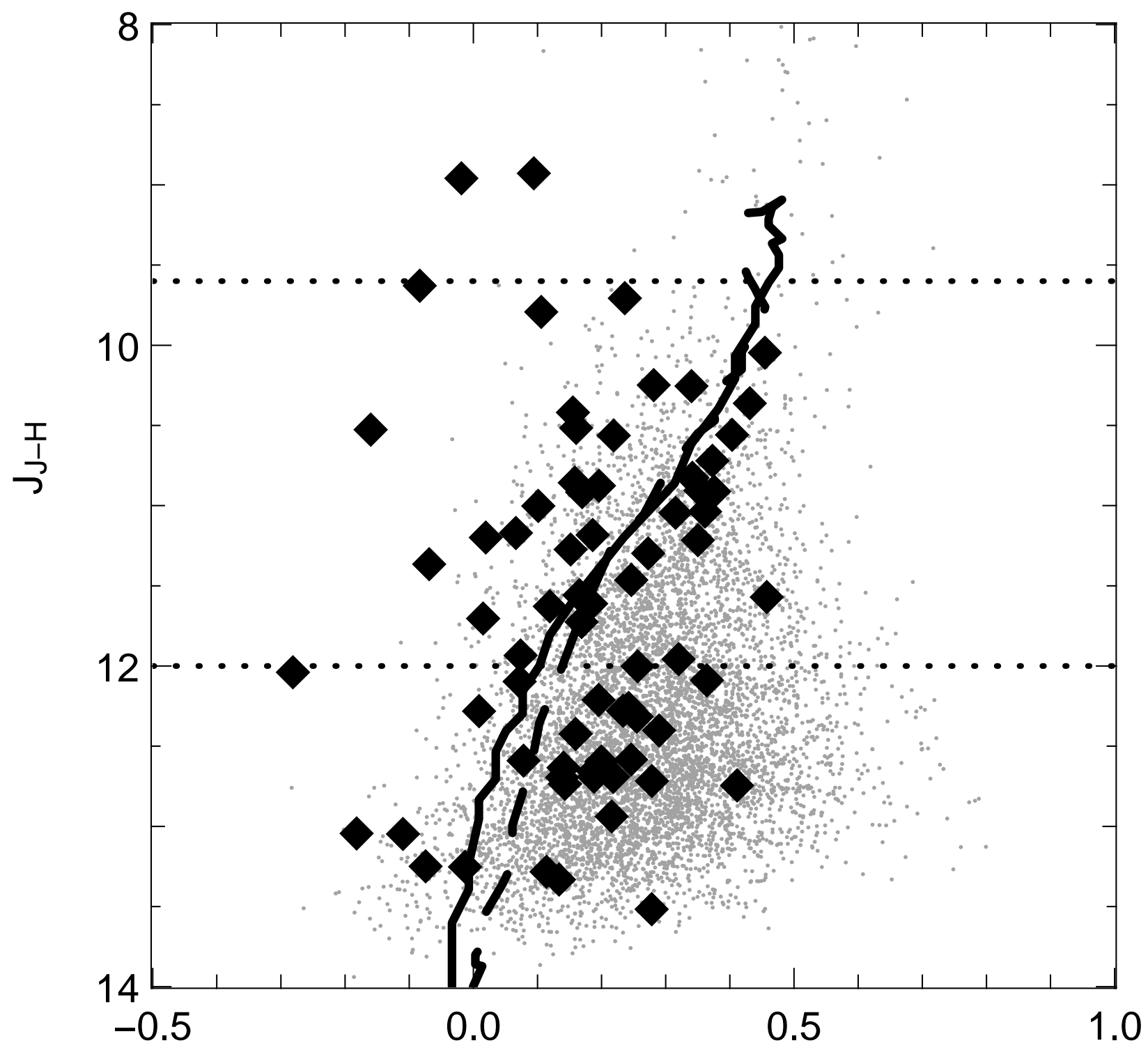

\section{QJHK}

Fig. 3.- Near-IR color-magnitude diagram for Orion. The reddening-invariant $\left(Q_{J H K}, J_{J-H}\right)$ color-magnitude diagram for the Orion sample is plotted here with the BCAH98 isochrones for Orion OB1a (dashed line) and Orion OB1b (solid line) shown as references. The reddening-invariant near-IR excess, $\Delta Q_{J H K}$, is measured relative to the isochrones for stars having $9.6<J_{J-H}<12.0$ (dotted lines). Disk candidates $\left(\Delta Q_{J H K}<0\right.$ ) are found to the left of the isochrones. The CTTS candidates, as inferred by SDSS multiband variability (see Figures 4 and 5), are highlighted by diamonds. 


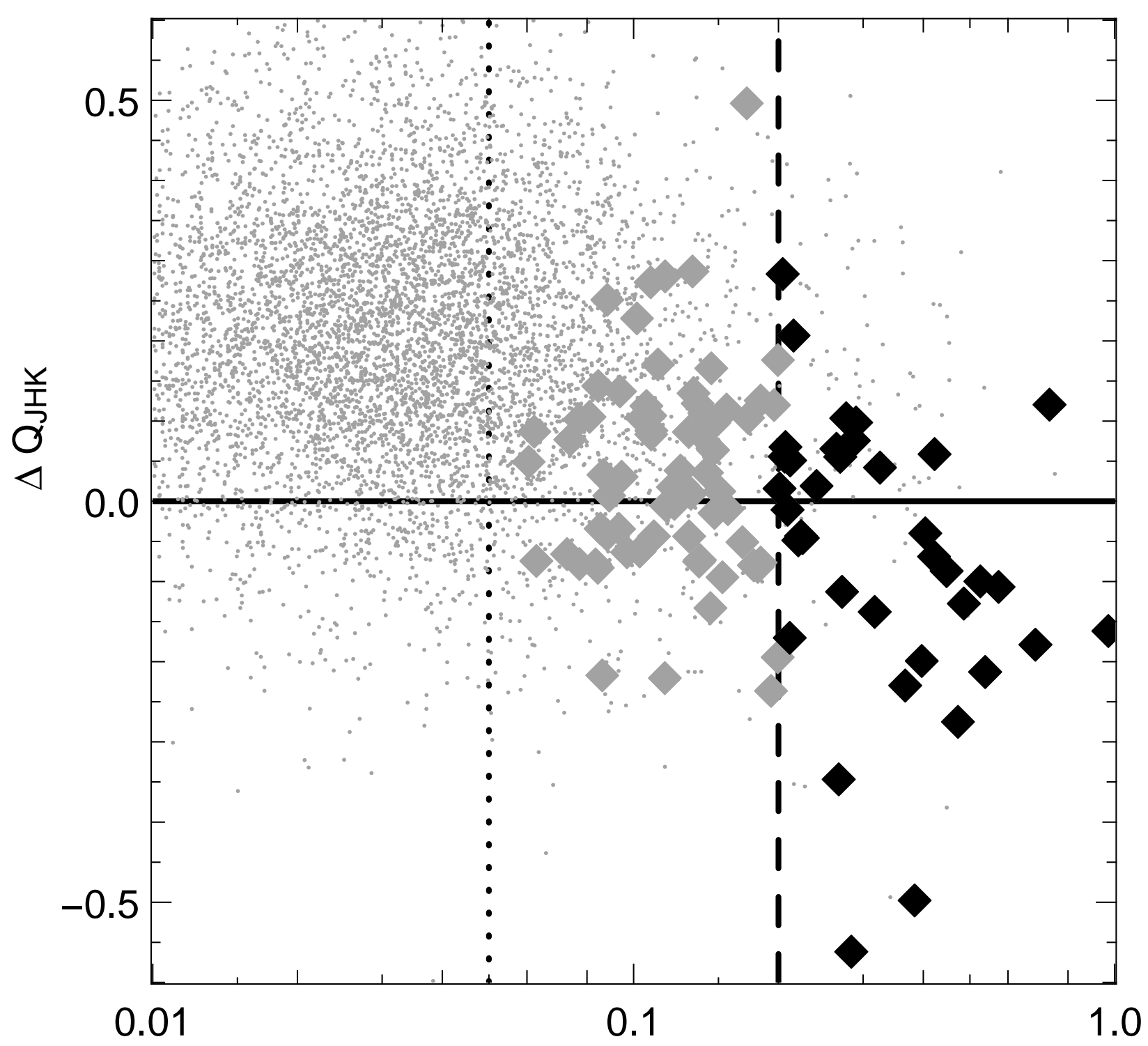

$\sigma_{g}$

Fig. 4.- Near-IR excess and $g$ band variability. In this figure we compare the intrinsic near-IR excess, as inferred by $\Delta Q_{J H K}$, and $\sigma_{g}$, the standard deviation in the $g$ band measured over all observations of a star. Stars meeting the variability thresholds of McGehee et al. (2005) for CTTS candidates $\left(\sigma_{g}>0.05\right.$ (dotted line) and $\sigma_{z}>0.05$ ) having $9.6<J_{J-H}<$ 12.0 (see Figure 3) are shown as diamonds. In this work we increase the $\sigma_{g}$ threshold to 0.2 magnitudes (dashed line) due to the marked increase in near-IR excess at this variability level and above. The stars meeting the new variability criteria of $\sigma_{g}>0.2$ and $\sigma_{z}>0.05$ are shown as black diamonds. 


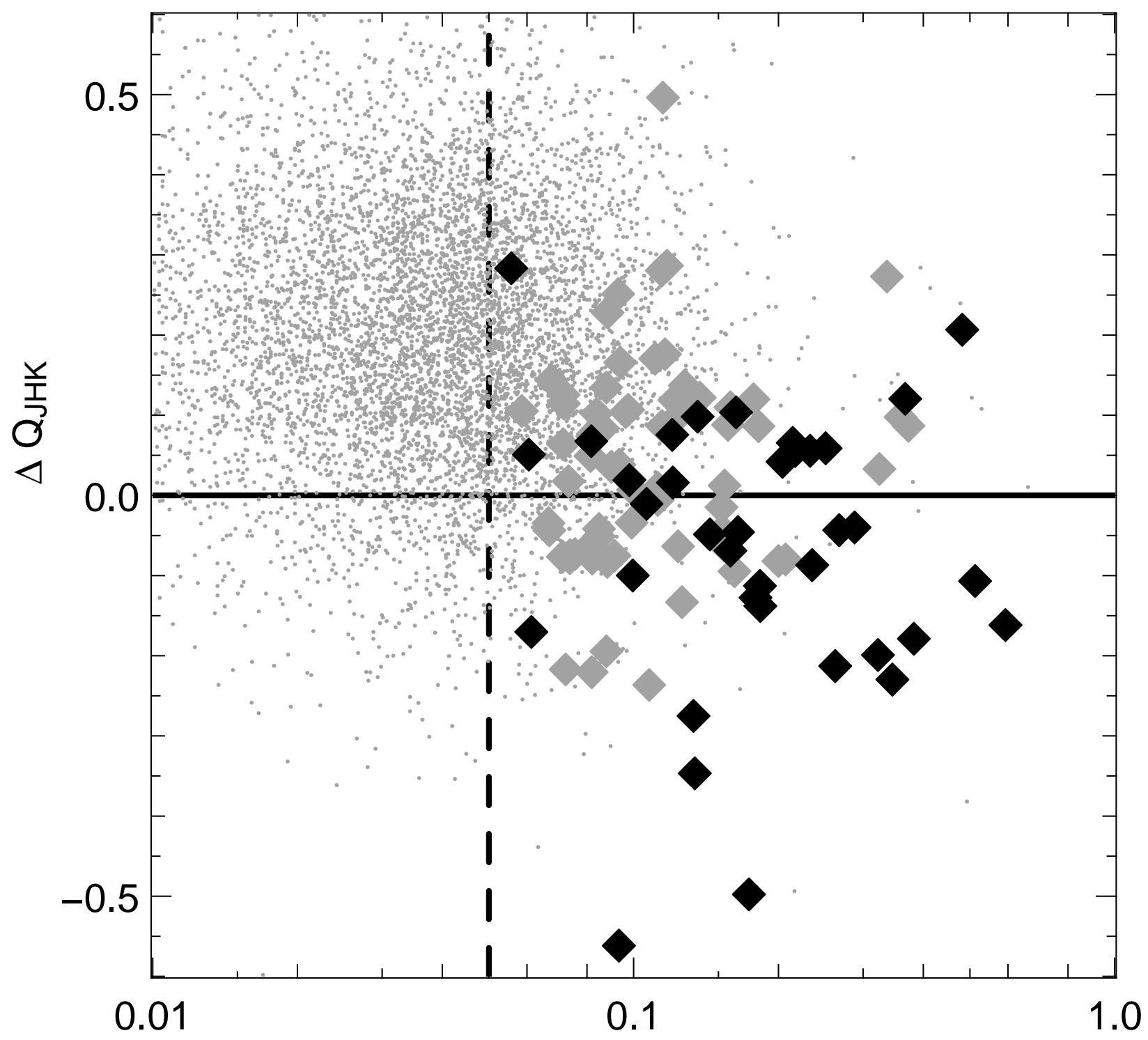

$\sigma_{\mathrm{Z}}$

Fig. 5.- Near-IR excess and $z$ band variability. Following Figure 4, we compare the intrinsic near-IR excess, as indicated by $\Delta Q_{J H K}$, and $\sigma_{z}$, the standard deviation in the $z$ band measured over all observations of a star. The $\sigma_{z}=0.05$ threshold used by McGehee et al. (2005) and this work is marked by the dashed line. As in Figure 4 the diamonds indicate CTTS candidates based on the criteria in McGehee et al. (2005) (grey) and this work (black). 


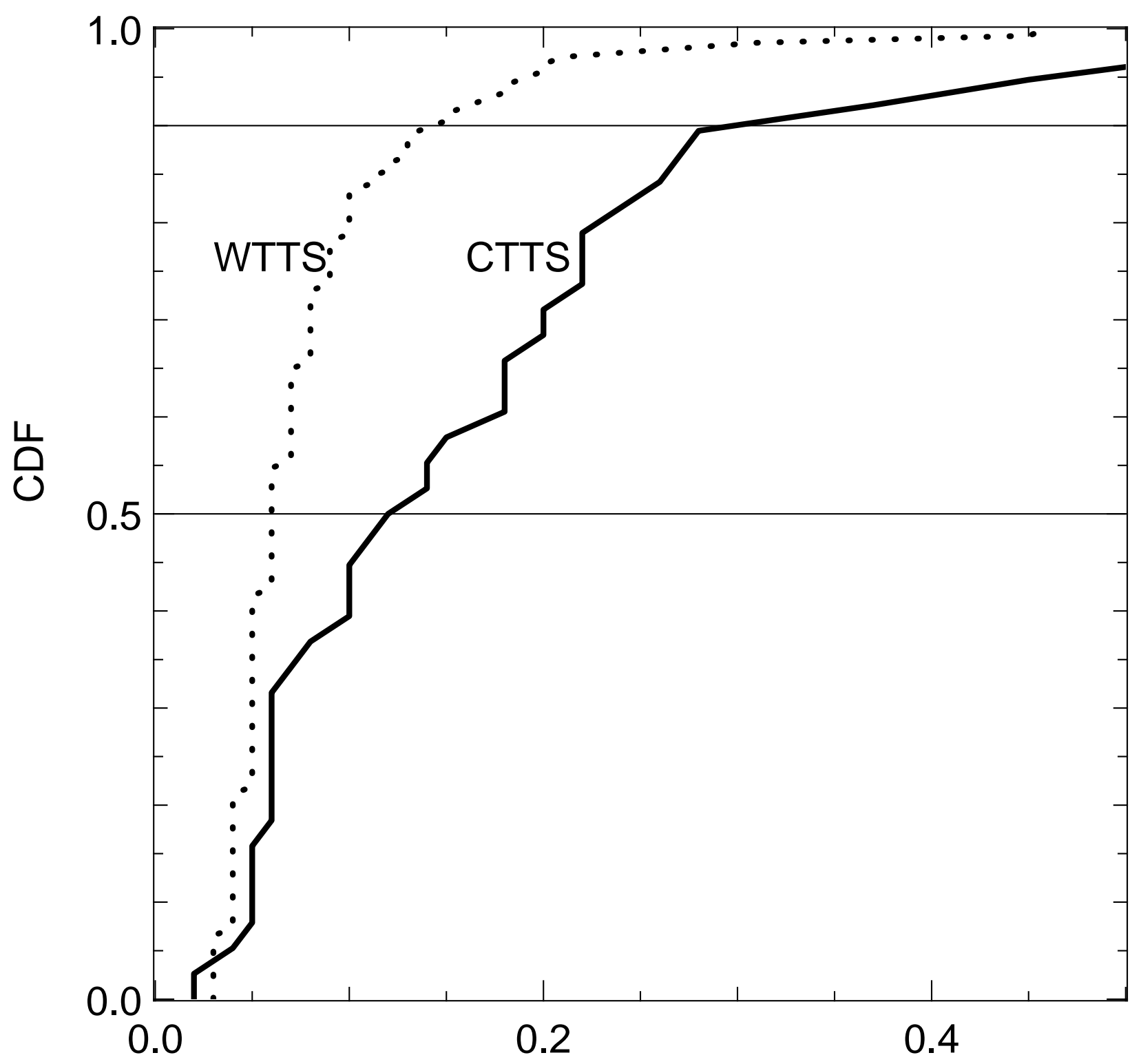

$\sigma \mathrm{V}$

Fig. 6.- $\sigma_{V}$ Cumulative Distribution Functions for WTTS and CTTS. The Cumulative Distribution Functions (CDF) of $\sigma_{V}$ are shown here for the spectroscopically confirmed WTTS (dotted line) and CTTS (solid line)) from Briceño et al. (2005b) where it is clear that the CTTS are much more highly variable than the WTTS. The median value of $\sigma_{V}$, at $\mathrm{CDF}=0.5$, for the WTTS and CTTS are 0.06 and 0.12 magnitudes, respectively. The 90th percentile values of $\sigma_{V}$ for the WTTS and CTTS have a greater spread of 0.14 magnitudes. 


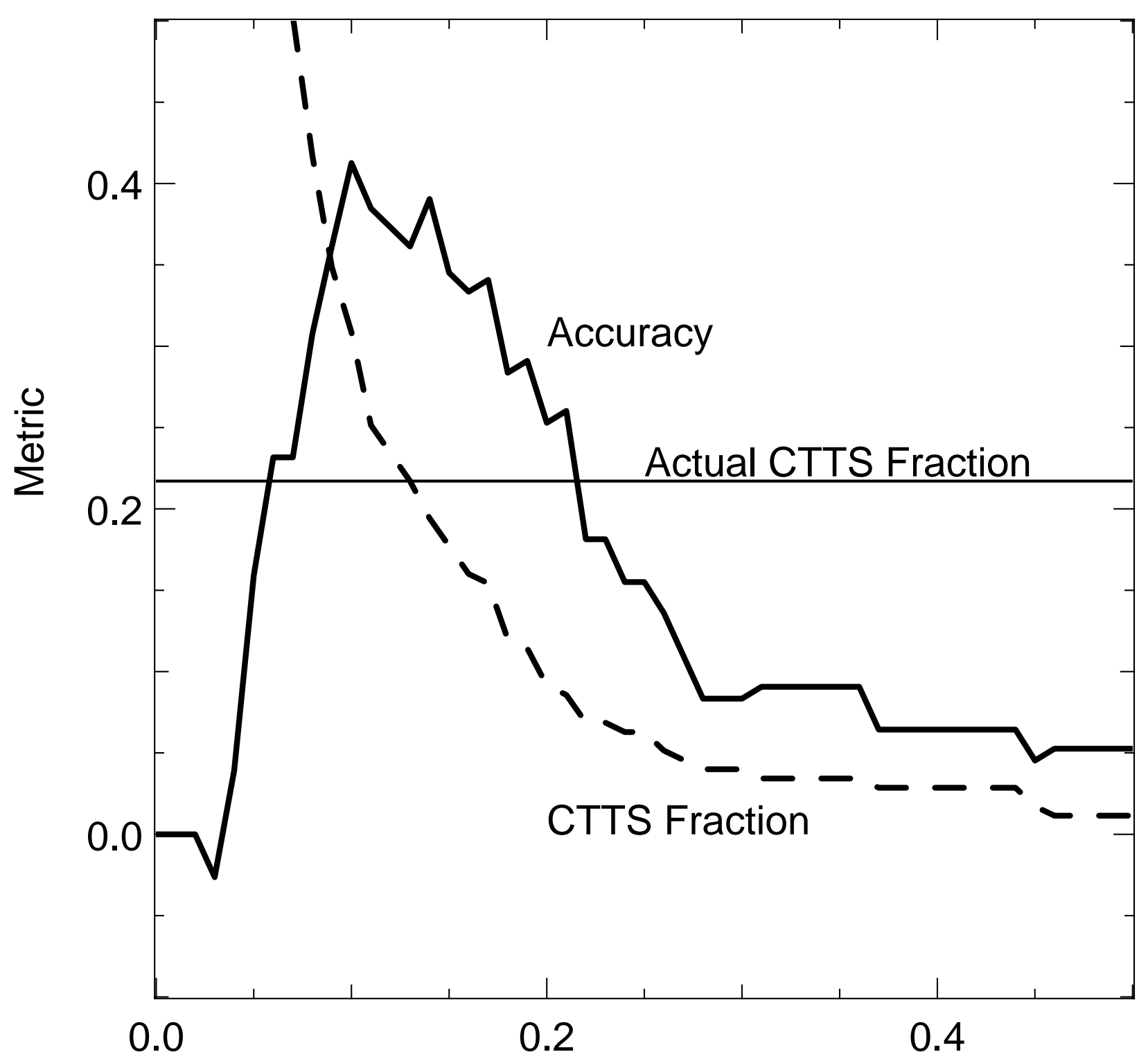

\section{$\sigma \mathrm{V}$}

Fig. 7.- Selection metrics for CTTS classification. Two selection metrics for CTTS/WTTS discriminations based on a threshold $\sigma_{V}$ value are compared here. To assess the ability to correct identify individual objects we plot the accuracy against the threshold (solid line). The dashed line shows the computed CTTS fraction (dashed line) which is compared against the actual value in the sample (horizontal line). Both selection metrics give their best performance for $0.10<\sigma_{V}<0.15$. 


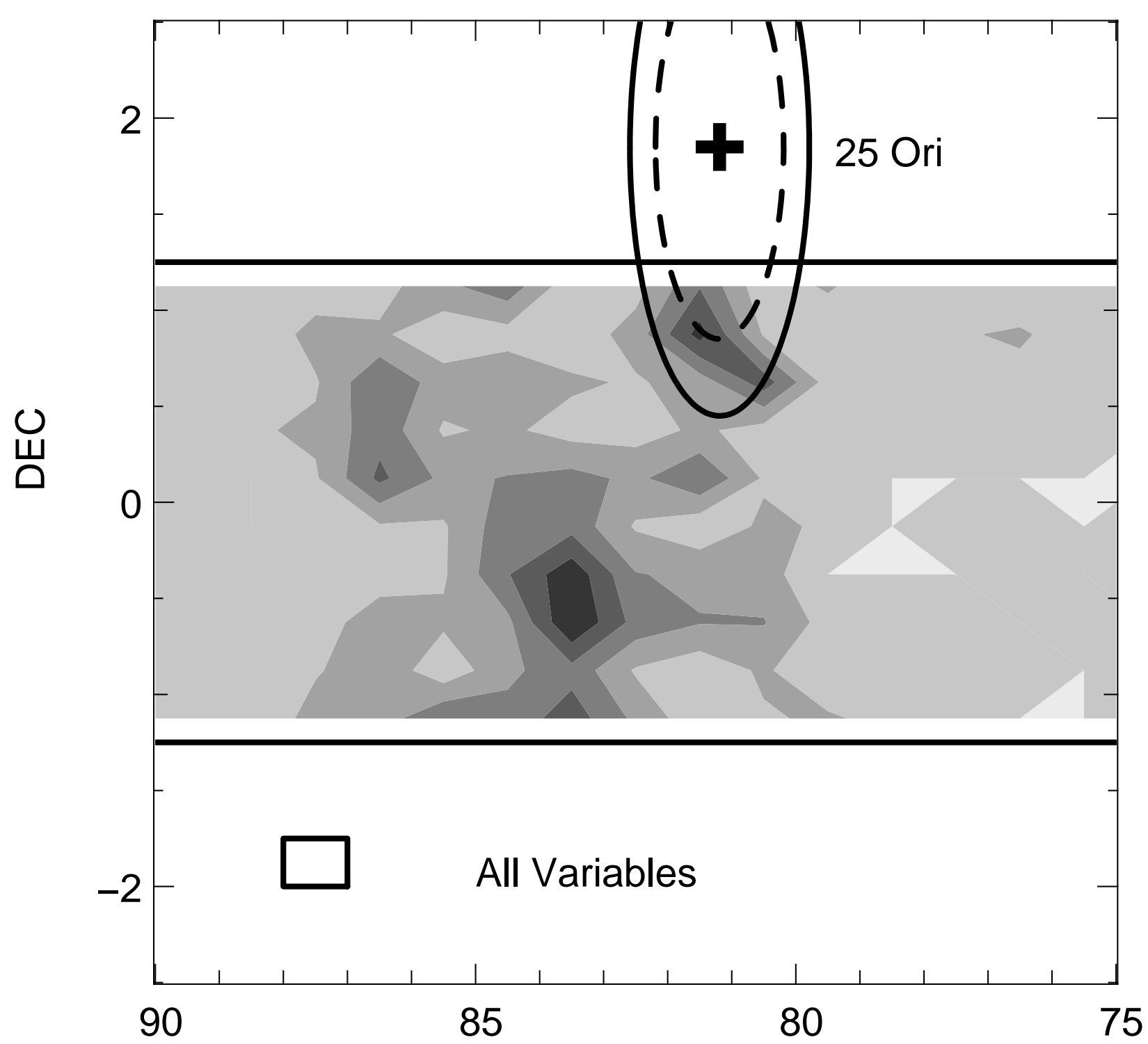

RA

Fig. 8. - Stellar densities. The surface densities of PMS candidates selected by variability $\left(\sigma_{g}>0.05\right)$ are presented here. The densities are computed based on $1.0^{\circ}$ by $0.25^{\circ}$ regions (see box in lower left) with the contours uniformly spaced between 0.0 stars $\operatorname{deg}^{-2}$ and a

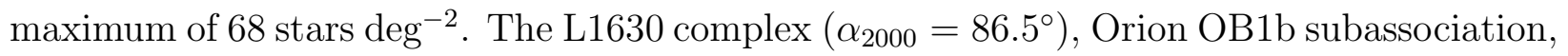
and the 25 Ori group are evident. The circles of radii $1.0^{\circ}$ and $1.4^{\circ}$ are centered on the Be star 25 Orionis (cross) and mark the approximate extent of the 25 Ori group. 


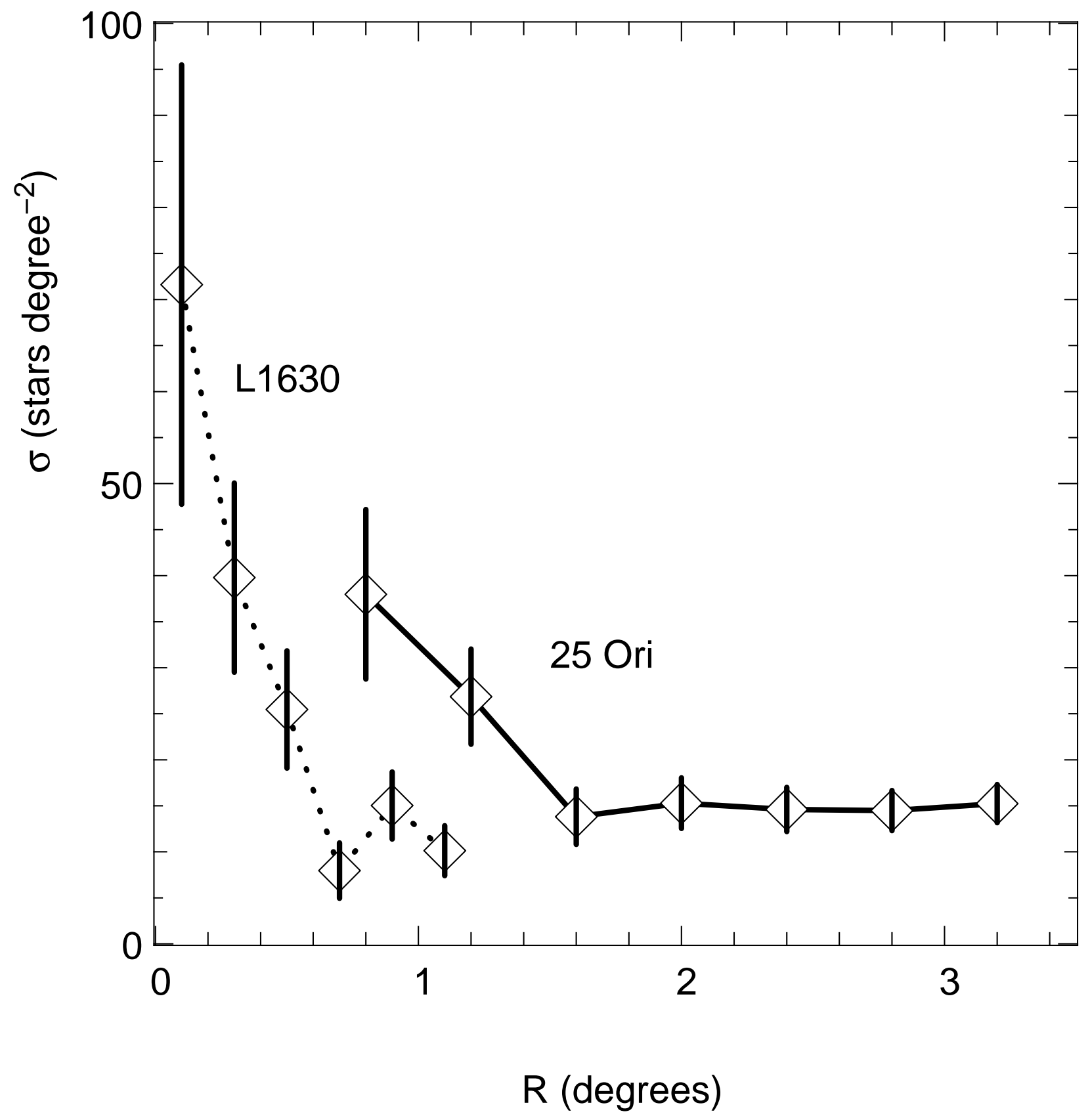

Fig. 9.- Stellar density versus distance from group center. The signatures of the 25 Ori group (solid line) and the NGC 2068/NGC 2071 active star formation site in L1630 (dotted line) are evident in this plot of the surface density of SDSS PMS candidates using radial bins. Spacings of $0.4^{\circ}$ and $0.2^{\circ}$ are used for the 25 Ori and L1630 regions, respectively. The innermost bin for the 25 Ori group spans distances of $0.6^{\circ}$ to $1.0^{\circ}$. We estimate the outer radius of the 25 Ori group as $1.4^{\circ}$ and that of the northern L1630 protocluster as $0.6^{\circ}$. The $\pm 1 \sigma$ error bars are computed based on Poisson statistics. 


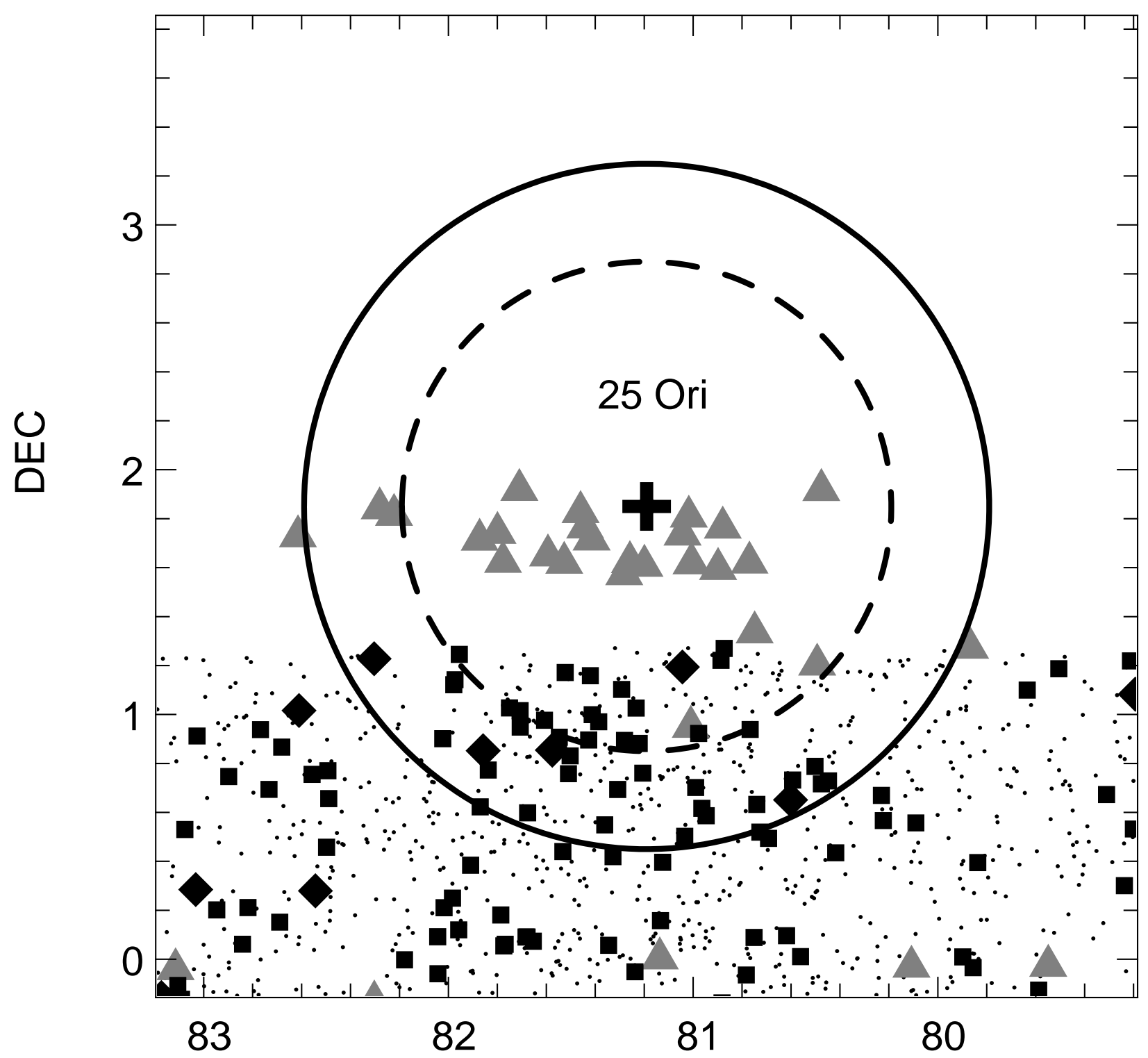

RA

Fig. 10.- The 25 Ori Region. In this figure we show the locations in equatorial coordinates of the spectroscopically confirmed T Tauris from the CIDA Variability Survey of Orion (grey triangles), SDSS WTTS candidates (small squares), and SDSS CTTS candidates (diamonds) within 2 degrees of 25 Ori. The circles of radii $1.0^{\circ}$ and $1.4^{\circ}$ are centered on the Be star 25 Ori (cross) and mark the approximate extent of the 25 Ori group. The candidate low-mass stars observed for least 2 epochs by the SDSS are shown as points. 


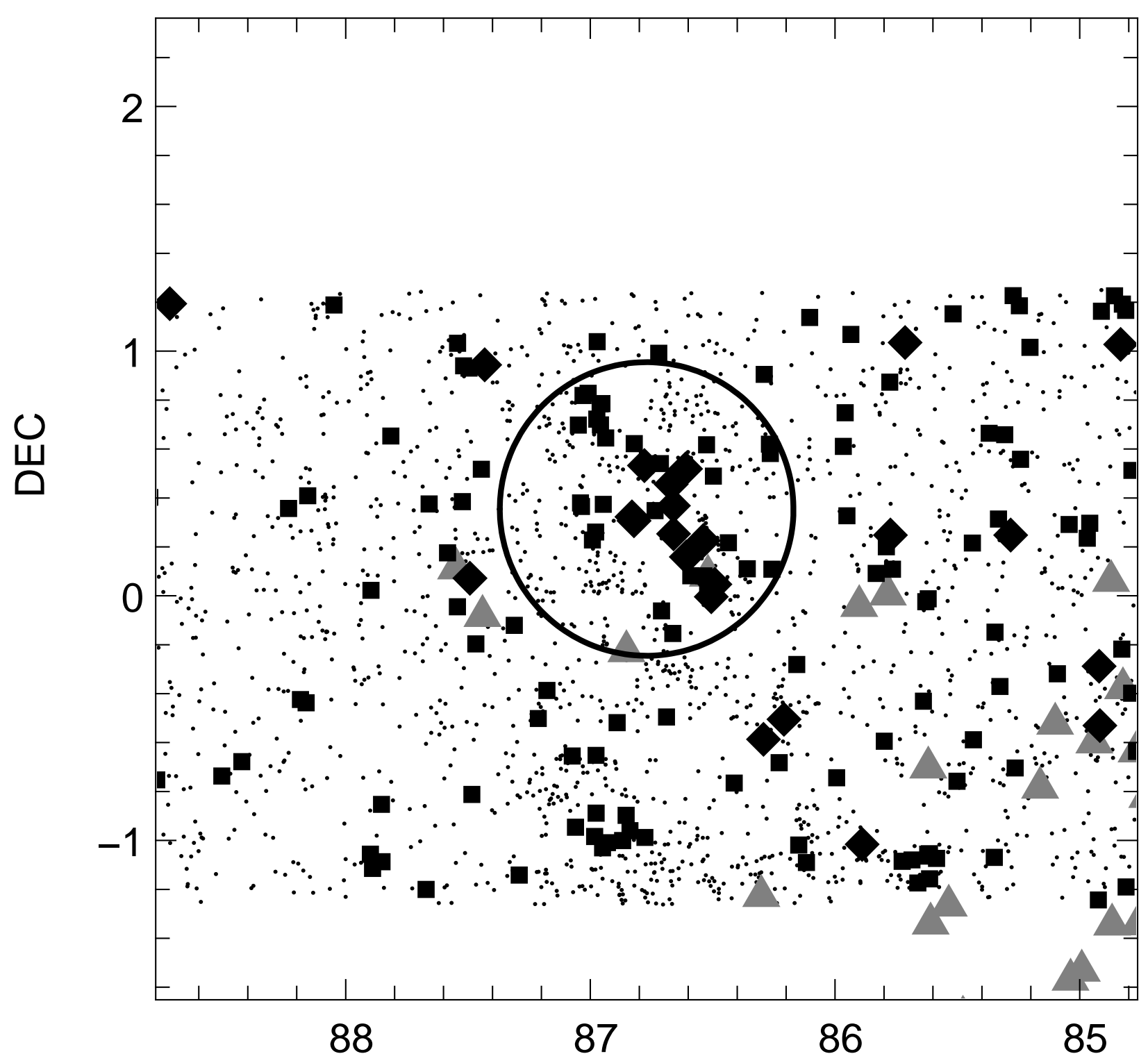

RA

Fig. 11. - The NGC 2068/NGC 2071 region. In this figure we show the locations in equatorial coordinates of the spectroscopically confirmed T Tauris from the CIDA Variability Survey of Orion (grey triangles), SDSS WTTS candidates (small squares), and SDSS CTTS candidates (diamonds) within 2 degrees of NGC 2071. The circle centered on NGC 2071 has a radius $0.6^{\circ}$. The candidate low-mass stars observed for least 2 epochs by the SDSS are shown as points. 


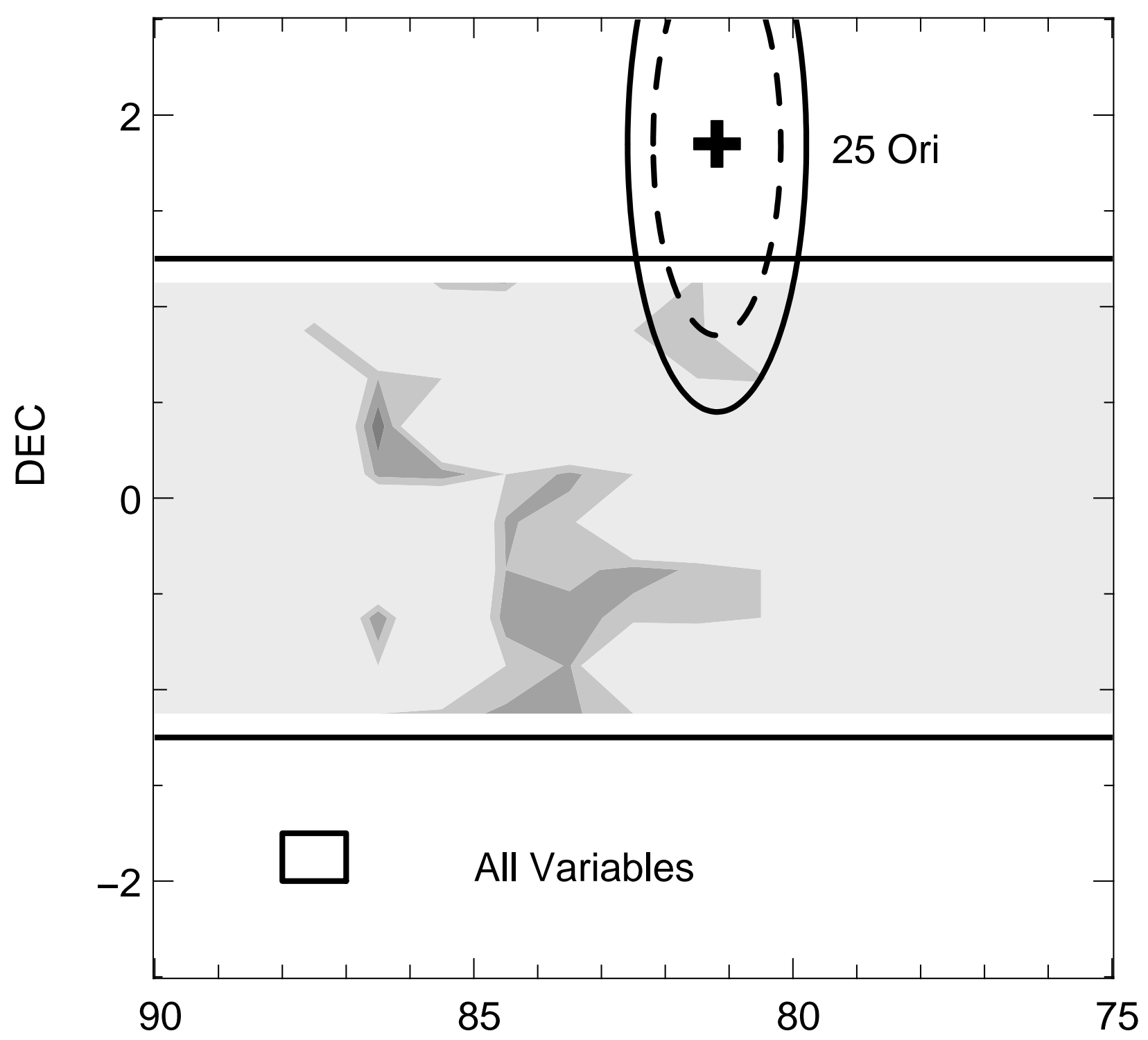

\section{RA}

Fig. 12.- CTTS fractions. The fraction of CTTS candidates, is shown here using the same samples and gridding as define in Figure 8. The contours are uniformly spaced between 0.0 and 1.0. Both the L1630 complex and the Orion OB1b subassociation have relatively high CTTS fractions. 


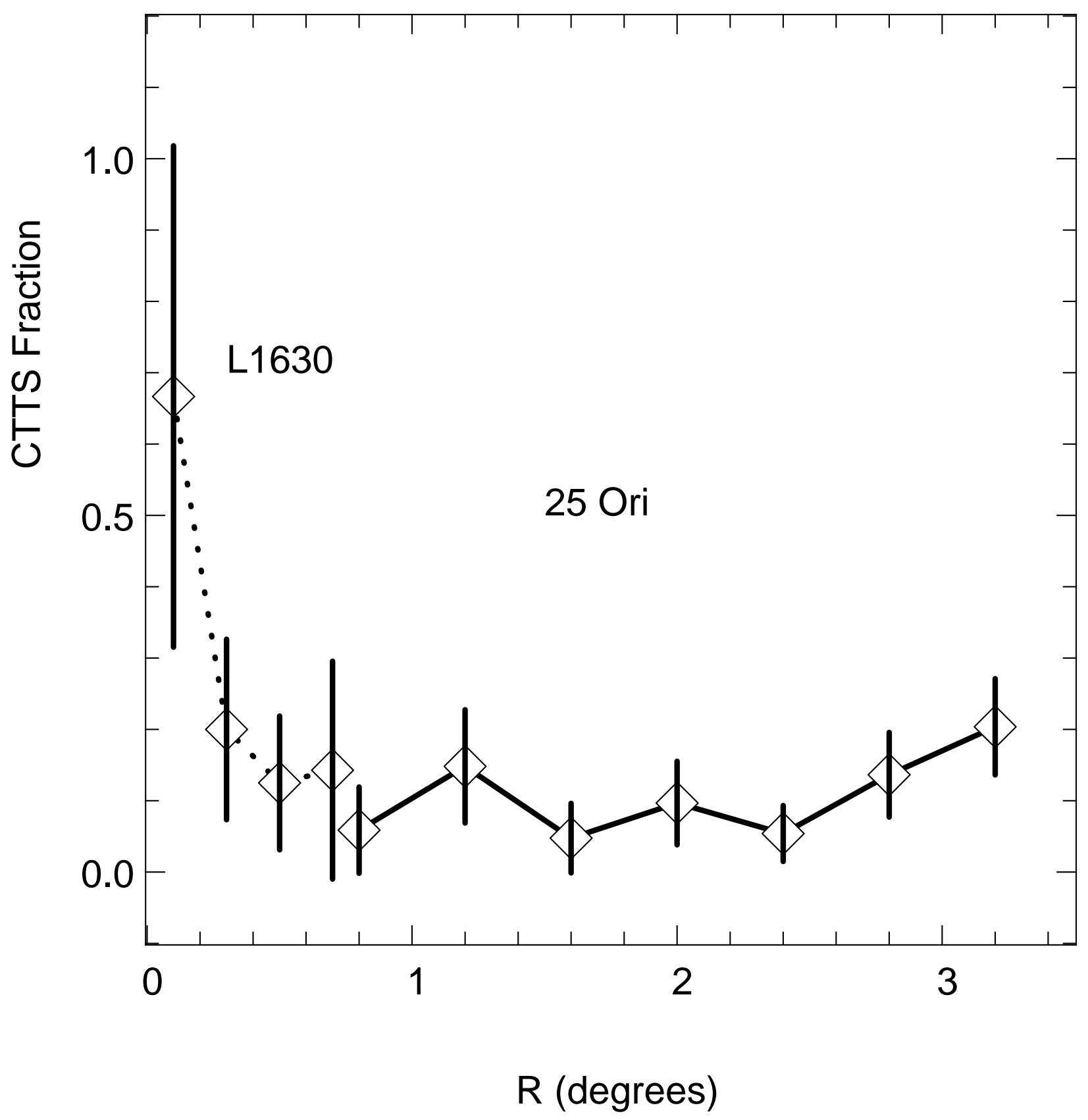

Fig. 13.- CTTS fraction versus distance from group center. The 25 Ori group has a mean CTTS fraction (solid line) as $\sim 0.1$, as seen in this plot of CTTS fraction against radial distance from 25 Orionis. This fraction is identical to that observed in the Orion OB1a field surrounding the 25 Ori group. In contrast, the central region of NGC 2068/NGC 2071 protocluster (dotted line) has a high CTTS fraction of $\sim 0.7$. The CTTS fractions are computed using the same radial bins as in Figure 9. The $\pm 1 \sigma$ error bars are computed based on Poisson statistics. 
Table 1. Matches between the SDSS and CVSO Surveys

\begin{tabular}{|c|c|c|c|c|c|c|c|c|}
\hline CVSO & SDSS Name & $g$ & $r$ & $i$ & $z$ & $\mathrm{R}^{\mathrm{a}}$ & Class $^{b}$ & Confirmed Class ${ }^{\mathrm{c}}$ \\
\hline CVSO 1 & SDSS J051058.78-000803.1 & 17.96 & 16.58 & 15.12 & 14.33 & 3.98 & $\mathrm{~W}$ & $\mathrm{C}$ \\
\hline CVSO 13 & SDSS J052242.82-005306.5 & 17.17 & 15.71 & 14.70 & 14.07 & 2.78 & $\mathrm{~W}$ & $\mathrm{~W}$ \\
\hline CVSO 49 & SDSS J052806.91-001806.0 & 18.47 & 17.06 & 15.74 & 14.98 & 2.31 & $\mathrm{~W}$ & $\mathrm{~W}$ \\
\hline CVSO 81 & SDSS J053055.62-005540.0 & 17.02 & 15.69 & 14.51 & 13.74 & 3.18 & - & $\mathrm{W}$ \\
\hline CVSO 124 & SDSS J053349.04-004625.9 & 16.81 & 15.44 & 14.29 & 13.63 & 3.47 & $\mathrm{~W}$ & $\mathrm{~W}$ \\
\hline CVSO 142 & SDSS J053530.03-002822.7 & 17.04 & 15.60 & 14.48 & 13.93 & 3.55 & - & $\mathrm{W}$ \\
\hline CVSO 156 & SDSS J053747.02-002007.2 & 16.73 & 15.38 & 14.25 & 13.50 & 3.92 & - & $\mathrm{C}$ \\
\hline CVSO 162 & SDSS J053830.84-011024.3 & 16.74 & 15.36 & 14.51 & 14.05 & 4.58 & - & $\mathrm{W}$ \\
\hline CVSO 164 & SDSS J053853.84-004920.0 & 17.51 & 16.14 & 14.64 & 13.56 & 4.43 & $\mathrm{~W}$ & $\mathrm{C}$ \\
\hline CVSO 168 & SDSS J053904.29-003810.0 & 18.19 & 16.76 & 15.34 & 14.48 & 4.36 & $\mathrm{~W}$ & $\mathrm{~W}$ \\
\hline
\end{tabular}

${ }^{a}$ Distance in degrees from the Be star 25 Orionis.

${ }^{\mathrm{b}}$ Preliminary classification as not PMS (-), WTTS (W) or CTTS (C) based on multi-band variability.

${ }^{\mathrm{c}}$ Classification as WTTS (W) or CTTS (C) on the basis of optical spectroscopy (Briceño et al. 2005b). 
Table 2. Candidate Low-Mass PMS in the Orion Equatorial Region

\begin{tabular}{|c|c|c|c|c|c|c|c|c|}
\hline Name & RA & DEC & $g$ & $r$ & $i$ & $z$ & $\mathrm{R}^{\mathrm{b}}$ & Class $^{\mathrm{c}}$ \\
\hline SDSS J050013.47+011349.1 & 75.0562 & 1.2303 & 19.90 & 18.24 & 16.67 & 15.77 & 6.17 & $\mathrm{~W}$ \\
\hline SDSS J050013.86+005807.4 & 75.0578 & 0.9687 & 16.85 & 15.44 & 14.57 & 14.09 & 6.20 & $\mathrm{~W}$ \\
\hline SDSS J050058.65+002106.9 & 75.2444 & 0.3519 & 19.92 & 18.29 & 16.14 & 15.03 & 6.13 & $\mathrm{~W}$ \\
\hline SDSS J050333.53-004138.2 & 75.8897 & -0.6940 & 18.79 & 17.40 & 15.84 & 14.96 & 5.88 & $\mathrm{~W}$ \\
\hline SDSS J050531.61+002616.3 & 76.3817 & 0.4379 & 20.56 & 18.78 & 16.82 & 15.88 & 5.01 & $\mathrm{~W}$ \\
\hline SDSS J050548.68-000332.8 & 76.4528 & -0.0591 & 19.06 & 17.33 & 16.35 & 15.86 & 5.11 & $\mathrm{~W}$ \\
\hline SDSS J050549.25+005120.9 & 76.4552 & 0.8558 & 19.22 & 17.54 & 16.50 & 15.94 & 4.84 & $\mathrm{~W}$ \\
\hline SDSS J050601.35-004831.0 & 76.5057 & -0.8086 & 18.14 & 16.56 & 15.93 & 15.64 & 5.39 & $\mathrm{~W}$ \\
\hline SDSS J050606.25+004226.5 & 76.5261 & 0.7074 & 18.65 & 17.05 & 15.85 & 15.28 & 4.80 & $\mathrm{~W}$ \\
\hline SDSS J050611.43+004053.3 & 76.5476 & 0.6815 & 19.64 & 18.09 & 16.87 & 16.29 & 4.79 & $\mathrm{~W}$ \\
\hline SDSS J050613.73+001627.2 & 76.5572 & 0.2742 & 18.58 & 17.03 & 15.98 & 15.47 & 4.89 & $\mathrm{~W}$ \\
\hline SDSS J050627.87-002129.1 & 76.6162 & -0.3581 & 17.66 & 16.26 & 15.12 & 14.45 & 5.08 & $\mathrm{~W}$ \\
\hline SDSS J050633.48+004747.3 & 76.6395 & 0.7965 & 19.25 & 17.70 & 16.50 & 15.88 & 4.67 & $\mathrm{~W}$ \\
\hline SDSS J050635.51-005531.6 & 76.6480 & -0.9255 & 18.83 & 17.32 & 15.86 & 15.11 & 5.32 & $\mathrm{~W}$ \\
\hline SDSS J050639.89-004913.1 & 76.6662 & -0.8203 & 16.27 & 14.70 & 13.38 & 12.74 & 5.25 & $\mathrm{~W}$ \\
\hline SDSS J050648.67+001748.6 & 76.7028 & 0.2968 & 18.93 & 17.30 & 16.16 & 15.73 & 4.75 & $\mathrm{~W}$ \\
\hline SDSS J050649.68-000917.4 & 76.7070 & -0.1549 & 18.07 & 16.64 & 15.49 & 14.92 & 4.91 & $\mathrm{~W}$ \\
\hline SDSS J050651.84+004813.6 & 76.7160 & 0.8038 & 18.99 & 17.45 & 16.34 & 15.81 & 4.59 & $\mathrm{~W}$ \\
\hline SDSS J050750.43+005414.9 & 76.9602 & 0.9042 & 18.44 & 17.07 & 15.79 & 15.11 & 4.33 & $\mathrm{~W}$ \\
\hline SDSS J050909.42+001512.5 & 77.2893 & 0.2535 & 18.58 & 17.26 & 16.27 & 15.78 & 4.21 & $\mathrm{C}$ \\
\hline SDSS J050910.93-005715.4 & 77.2956 & -0.9543 & 18.30 & 16.89 & 15.59 & 14.85 & 4.80 & $\mathrm{~W}$ \\
\hline SDSS J050939.12-003739.0 & 77.4130 & -0.6275 & 18.35 & 16.83 & 15.51 & 14.59 & 4.52 & $\mathrm{~W}$ \\
\hline SDSS J051037.47+004943.4 & 77.6561 & 0.8287 & 17.61 & 16.59 & 15.32 & 14.99 & 3.68 & $\mathrm{~W}$ \\
\hline SDSS J051052.52-003700.4 & 77.7188 & -0.6168 & 19.47 & 18.28 & 16.95 & 16.03 & 4.26 & $\mathrm{C}$ \\
\hline SDSS J051058.78-000803.1 & 77.7449 & -0.1342 & 17.96 & 16.58 & 15.12 & 14.33 & 3.98 & $\mathrm{~W}$ \\
\hline SDSS J051107.89+005450.4 & 77.7829 & 0.9140 & 19.54 & 18.11 & 16.75 & 16.00 & 3.53 & $\mathrm{~W}$ \\
\hline SDSS J051112.40+005645.5 & 77.8017 & 0.9460 & 17.29 & 15.88 & 14.23 & 13.27 & 3.51 & $\mathrm{~W}$ \\
\hline SDSS J051142.74+010149.1 & 77.9281 & 1.0303 & 18.42 & 17.04 & 15.72 & 15.00 & 3.36 & $\mathrm{~W}$ \\
\hline SDSS J051232.90-003851.5 & 78.1371 & -0.6477 & 19.79 & 18.35 & 16.90 & 16.02 & 3.94 & $\mathrm{~W}$ \\
\hline SDSS J051313.77+001702.9 & 78.3074 & 0.2842 & 18.27 & 16.87 & 15.65 & 15.03 & 3.28 & $\mathrm{~W}$ \\
\hline SDSS J051347.73+005505.5 & 78.4489 & 0.9182 & 16.87 & 15.36 & 14.22 & 13.59 & 2.90 & $\mathrm{~W}$ \\
\hline SDSS J051351.57-011337.3 & 78.4649 & -1.2270 & 16.78 & 15.39 & 14.24 & 13.62 & 4.11 & $\mathrm{~W}$ \\
\hline SDSS J051445.52+004416.1 & 78.6897 & 0.7378 & 19.29 & 17.63 & 16.38 & 15.78 & 2.74 & $\mathrm{~W}$ \\
\hline SDSS J051454.97+004640.2 & 78.7291 & 0.7779 & 18.65 & 17.01 & 15.72 & 15.07 & 2.68 & $\mathrm{~W}$ \\
\hline SDSS J051515.78-010006.4 & 78.8158 & -1.0018 & 19.24 & 17.66 & 16.75 & 16.22 & 3.71 & $\mathrm{~W}$ \\
\hline SDSS J051518.75+004333.4 & 78.8281 & 0.7260 & 16.84 & 15.29 & 14.59 & 14.26 & 2.62 & $\mathrm{~W}$ \\
\hline SDSS J051646.31+010458.7 & 79.1930 & 1.0830 & 19.92 & 18.54 & 17.13 & 16.33 & 2.14 & $\mathrm{C}$ \\
\hline SDSS J051648.18+003155.9 & 79.2008 & 0.5322 & 17.86 & 16.41 & 15.33 & 14.74 & 2.39 & $\mathrm{~W}$ \\
\hline SDSS J051651.05+011308.1 & 79.2127 & 1.2189 & 18.51 & 17.15 & 15.69 & 14.88 & 2.08 & $\mathrm{~W}$ \\
\hline SDSS J051657.06+001802.0 & 79.2378 & 0.3006 & 19.83 & 18.37 & 16.52 & 15.55 & 2.49 & $\mathrm{~W}$ \\
\hline SDSS J051714.49+004023.1 & 79.3104 & 0.6731 & 17.84 & 16.42 & 14.95 & 14.23 & 2.22 & $\mathrm{~W}$ \\
\hline SDSS J051755.36-003912.7 & 79.4807 & -0.6535 & 17.68 & 16.25 & 15.15 & 14.51 & 3.03 & $\mathrm{C}$ \\
\hline SDSS J051801.35+011112.1 & 79.5057 & 1.1867 & 17.88 & 16.46 & 15.50 & 15.03 & 1.81 & $\mathrm{~W}$ \\
\hline SDSS J051809.05-001230.7 & 79.5377 & -0.2086 & 19.33 & 17.93 & 16.39 & 15.59 & 2.64 & $\mathrm{~W}$ \\
\hline SDSS J051821.11-000733.6 & 79.5880 & -0.1260 & 17.80 & 16.38 & 15.20 & 14.51 & 2.54 & $\mathrm{~W}$ \\
\hline SDSS J051822.37-010511.0 & 79.5932 & -1.0864 & 19.03 & 17.57 & 16.04 & 15.15 & 3.34 & $\mathrm{~W}$ \\
\hline
\end{tabular}


Table 2-Continued

\begin{tabular}{|c|c|c|c|c|c|c|c|c|}
\hline Name & RA & DEC & $g$ & $r$ & $i$ & $z$ & $\mathrm{R}^{\mathrm{b}}$ & Class $^{\mathrm{C}}$ \\
\hline SDSS J051832.38+010559.4 & 79.6349 & 1.0998 & 18.14 & 16.66 & 15.72 & 15.17 & 1.73 & $\mathrm{~W}$ \\
\hline SDSS J051858.70-003815.6 & 79.7446 & -0.6377 & 18.78 & 17.35 & 15.90 & 14.94 & 2.88 & $\mathrm{~W}$ \\
\hline SDSS J051912.02-010514.9 & 79.8001 & -1.0875 & 17.15 & 15.75 & 14.79 & 14.26 & 3.25 & $\mathrm{C}$ \\
\hline SDSS J051920.84+002341.3 & 79.8369 & 0.3948 & 17.26 & 15.86 & 14.82 & 14.26 & 1.99 & W \\
\hline SDSS J051925.42-000203.0 & 79.8560 & -0.0342 & 19.16 & 17.91 & 16.55 & 15.89 & 2.31 & $\mathrm{~W}$ \\
\hline SDSS J051926.99-011157.1 & 79.8625 & -1.1992 & 20.45 & 18.97 & 17.43 & 16.53 & 3.33 & $\mathrm{C}$ \\
\hline SDSS J051935.47+000035.0 & 79.8978 & 0.0097 & 18.65 & 17.18 & 16.32 & 15.91 & 2.25 & $\mathrm{~W}$ \\
\hline SDSS J051947.40-011420.6 & 79.9475 & -1.2391 & 19.82 & 18.32 & 16.67 & 15.73 & 3.33 & W \\
\hline SDSS J052015.55-001922.2 & 80.0648 & -0.3229 & 21.37 & 19.86 & 17.89 & 16.79 & 2.45 & $\mathrm{~W}$ \\
\hline SDSS J052021.54+003325.1 & 80.0898 & 0.5570 & 19.79 & 18.38 & 17.11 & 16.37 & 1.70 & $\mathrm{~W}$ \\
\hline SDSS J052024.03-004631.9 & 80.1001 & -0.7755 & 19.22 & 17.79 & 16.23 & 15.20 & 2.84 & $\mathrm{~W}$ \\
\hline SDSS J052033.20-004330.2 & 80.1384 & -0.7251 & 19.37 & 17.94 & 16.53 & 15.78 & 2.78 & $\mathrm{~W}$ \\
\hline SDSS J052042.36-001723.9 & 80.1765 & -0.2900 & 19.60 & 18.13 & 16.94 & 16.28 & 2.37 & $\mathrm{~W}$ \\
\hline SDSS J052042.75-004309.6 & 80.1781 & -0.7194 & 18.90 & 17.37 & 15.94 & 15.01 & 2.76 & $\mathrm{~W}$ \\
\hline SDSS J052047.14-004840.3 & 80.1964 & -0.8112 & 18.37 & 16.85 & 16.11 & 15.78 & 2.84 & $\mathrm{~W}$ \\
\hline SDSS J052053.45+003359.3 & 80.2227 & 0.5665 & 17.83 & 16.43 & 15.22 & 14.54 & 1.61 & $\mathrm{~W}$ \\
\hline SDSS J052055.35+004009.8 & 80.2307 & 0.6694 & 19.17 & 17.66 & 15.89 & 14.93 & 1.52 & W \\
\hline SDSS J052139.86-004454.2 & 80.4161 & -0.7484 & 18.39 & 16.88 & 15.55 & 14.77 & 2.71 & $\mathrm{~W}$ \\
\hline SDSS J052140.09+002605.9 & 80.4171 & 0.4350 & 17.88 & 16.46 & 15.29 & 14.63 & 1.61 & $\mathrm{~W}$ \\
\hline SDSS J052147.21+004343.0 & 80.4467 & 0.7286 & 16.36 & 14.92 & 14.17 & 13.89 & 1.35 & $\mathrm{~W}$ \\
\hline SDSS J052154.25+004259.3 & 80.4761 & 0.7165 & 18.53 & 17.10 & 15.99 & 15.52 & 1.34 & W \\
\hline SDSS J052154.83-004323.3 & 80.4785 & -0.7231 & 18.13 & 16.68 & 15.57 & 14.91 & 2.67 & $\mathrm{~W}$ \\
\hline SDSS J052200.52+004716.3 & 80.5022 & 0.7879 & 20.26 & 18.67 & 17.18 & 16.36 & 1.27 & $\mathrm{~W}$ \\
\hline SDSS J052214.62+000039.5 & 80.5610 & 0.0110 & 17.84 & 16.31 & 15.47 & 15.06 & 1.94 & W \\
\hline SDSS J052217.23-001611.0 & 80.5718 & -0.2697 & 18.03 & 16.63 & 15.55 & 14.94 & 2.21 & $\mathrm{~W}$ \\
\hline SDSS J052222.44+004356.4 & 80.5935 & 0.7323 & 18.09 & 16.61 & 15.18 & 14.46 & 1.27 & W \\
\hline SDSS J052224.45+003903.7 & 80.6019 & 0.6511 & 19.84 & 18.35 & 16.65 & 15.80 & 1.34 & $\mathrm{C}$ \\
\hline SDSS J052224.91-001708.7 & 80.6038 & -0.2858 & 19.03 & 17.59 & 16.57 & 16.04 & 2.21 & $\mathrm{~W}$ \\
\hline SDSS J052228.40+000546.0 & 80.6183 & 0.0961 & 18.01 & 16.53 & 15.78 & 15.41 & 1.84 & $\mathrm{~W}$ \\
\hline SDSS J052230.85-001235.5 & 80.6286 & -0.2099 & 16.56 & 15.12 & 14.20 & 13.71 & 2.14 & W \\
\hline SDSS J052235.19-010846.7 & 80.6466 & -1.1463 & 17.90 & 16.47 & 15.26 & 14.53 & 3.05 & $\mathrm{C}$ \\
\hline SDSS J052242.82-005306.5 & 80.6784 & -0.8851 & 17.17 & 15.71 & 14.70 & 14.07 & 2.78 & $\mathrm{~W}$ \\
\hline SDSS J052245.99+002937.8 & 80.6916 & 0.4939 & 16.74 & 15.36 & 14.46 & 13.95 & 1.44 & $\mathrm{~W}$ \\
\hline SDSS J052254.52+003110.3 & 80.7272 & 0.5195 & 19.39 & 17.98 & 16.27 & 15.35 & 1.41 & $\mathrm{~W}$ \\
\hline SDSS J052256.90-003343.8 & 80.7371 & -0.5622 & 19.00 & 17.60 & 16.28 & 15.49 & 2.45 & W \\
\hline SDSS J052257.49+003757.6 & 80.7396 & 0.6327 & 18.64 & 17.18 & 15.64 & 14.82 & 1.30 & $\mathrm{~W}$ \\
\hline SDSS J052300.22+000520.5 & 80.7509 & 0.0890 & 18.07 & 16.69 & 15.25 & 14.52 & 1.81 & W \\
\hline SDSS J052304.07+005619.9 & 80.7670 & 0.9389 & 18.71 & 17.28 & 16.33 & 15.81 & 1.00 & $\mathrm{~W}$ \\
\hline SDSS J052305.37-004007.8 & 80.7724 & -0.6688 & 17.46 & 16.04 & 15.15 & 14.61 & 2.55 & W \\
\hline SDSS J052307.82-000351.0 & 80.7826 & -0.0642 & 19.69 & 18.24 & 16.84 & 16.13 & 1.96 & $\mathrm{~W}$ \\
\hline SDSS J052323.30-002713.6 & 80.8471 & -0.4538 & 17.64 & 16.20 & 14.99 & 14.36 & 2.33 & $\mathrm{~W}$ \\
\hline SDSS J052324.18-010319.3 & 80.8508 & -1.0554 & 18.98 & 17.49 & 16.63 & 16.22 & 2.93 & $\mathrm{~W}$ \\
\hline SDSS J052329.50+011612.2 & 80.8729 & 1.2701 & 17.15 & 15.71 & 14.53 & 13.83 & 0.66 & $\mathrm{~W}$ \\
\hline SDSS J052331.69-001042.3 & 80.8821 & -0.1784 & 19.57 & 18.19 & 16.53 & 15.56 & 2.05 & W \\
\hline SDSS J052332.70+011317.1 & 80.8863 & 1.2214 & 17.43 & 16.01 & 14.93 & 14.36 & 0.70 & $\mathrm{~W}$ \\
\hline SDSS J052338.33-001247.6 & 80.9097 & -0.2132 & 19.61 & 18.21 & 16.69 & 15.77 & 2.08 & W \\
\hline
\end{tabular}


Table 2-Continued

\begin{tabular}{|c|c|c|c|c|c|c|c|c|}
\hline Name & RA & DEC & $g$ & $r$ & $i$ & $z$ & $\mathrm{R}^{\mathrm{b}}$ & Class $^{\mathrm{C}}$ \\
\hline SDSS J052347.15+003510.5 & 80.9465 & 0.5863 & 18.79 & 17.39 & 15.89 & 15.12 & 1.29 & $\mathrm{~W}$ \\
\hline SDSS J052348.42-011350.4 & 80.9518 & -1.2307 & 19.28 & 17.78 & 16.10 & 15.10 & 3.09 & $\mathrm{~W}$ \\
\hline SDSS J052351.47+003700.6 & 80.9645 & 0.6168 & 17.74 & 16.27 & 15.01 & 14.40 & 1.25 & $\mathrm{~W}$ \\
\hline SDSS J052354.63+005520.8 & 80.9776 & 0.9225 & 16.86 & 15.44 & 14.61 & 14.15 & 0.95 & $\mathrm{~W}$ \\
\hline SDSS J052356.59-005814.7 & 80.9858 & -0.9708 & 17.34 & 15.92 & 14.64 & 13.98 & 2.83 & W \\
\hline SDSS J052356.65-004223.6 & 80.9861 & -0.7066 & 18.26 & 16.90 & 15.37 & 14.49 & 2.56 & $\mathrm{~W}$ \\
\hline SDSS J052357.49+004206.3 & 80.9896 & 0.7018 & 20.10 & 18.68 & 16.97 & 16.06 & 1.17 & $\mathrm{~W}$ \\
\hline SDSS J052403.31-001838.9 & 81.0138 & -0.3108 & 18.66 & 17.26 & 15.99 & 15.28 & 2.17 & $\mathrm{~W}$ \\
\hline SDSS J052408.21+003007.9 & 81.0342 & 0.5022 & 17.63 & 16.25 & 15.09 & 14.48 & 1.36 & $\mathrm{~W}$ \\
\hline SDSS J052410.49+011138.0 & 81.0437 & 1.1939 & 17.15 & 15.80 & 14.86 & 14.33 & 0.67 & $\mathrm{C}$ \\
\hline SDSS J052429.84+002345.9 & 81.1244 & 0.3961 & 17.64 & 16.40 & 15.16 & 14.35 & 1.46 & W \\
\hline SDSS J052432.01+000928.7 & 81.1334 & 0.1580 & 18.30 & 16.91 & 15.48 & 14.76 & 1.69 & W \\
\hline SDSS J052436.76-004246.7 & 81.1532 & -0.7130 & 20.04 & 18.58 & 16.97 & 16.07 & 2.56 & $\mathrm{~W}$ \\
\hline SDSS J052441.03-002631.4 & 81.1710 & -0.4421 & 18.53 & 17.05 & 15.78 & 15.07 & 2.29 & $\mathrm{~W}$ \\
\hline SDSS J052443.29-002144.6 & 81.1804 & -0.3624 & 18.77 & 17.31 & 16.33 & 15.77 & 2.21 & $\mathrm{~W}$ \\
\hline SDSS J052446.57-001632.1 & 81.1941 & -0.2756 & 18.65 & 17.25 & 15.91 & 15.15 & 2.13 & $\mathrm{~W}$ \\
\hline SDSS J052447.78-003741.4 & 81.1991 & -0.6282 & 19.41 & 18.00 & 16.38 & 15.49 & 2.48 & $\mathrm{~W}$ \\
\hline SDSS J052449.37+004537.3 & 81.2057 & 0.7604 & 17.34 & 15.77 & 14.83 & 14.23 & 1.09 & $\mathrm{~W}$ \\
\hline SDSS J052452.91+005254.1 & 81.2205 & 0.8817 & 19.83 & 18.38 & 17.03 & 16.30 & 0.97 & $\mathrm{~W}$ \\
\hline SDSS J052455.89+010133.8 & 81.2329 & 1.0261 & 20.90 & 19.35 & 17.30 & 16.18 & 0.83 & $\mathrm{~W}$ \\
\hline SDSS J052457.07-000304.1 & 81.2378 & -0.0512 & 16.83 & 15.45 & 14.51 & 13.96 & 1.90 & $\mathrm{~W}$ \\
\hline SDSS J052505.71-003030.7 & 81.2738 & -0.5085 & 20.48 & 19.05 & 17.38 & 16.37 & 2.36 & $\mathrm{C}$ \\
\hline SDSS J052507.32+005339.5 & 81.2805 & 0.8943 & 19.27 & 17.84 & 16.25 & 15.40 & 0.96 & $\mathrm{~W}$ \\
\hline SDSS J052510.11+010608.6 & 81.2922 & 1.1024 & 17.67 & 16.19 & 15.06 & 14.43 & 0.75 & $\mathrm{~W}$ \\
\hline SDSS J052513.93+004137.3 & 81.3081 & 0.6937 & 20.53 & 18.73 & 16.99 & 16.11 & 1.16 & $\mathrm{~W}$ \\
\hline SDSS J052514.52-005626.6 & 81.3105 & -0.9407 & 19.57 & 18.15 & 16.56 & 15.65 & 2.79 & $\mathrm{~W}$ \\
\hline SDSS J052518.59+002508.9 & 81.3275 & 0.4192 & 18.17 & 16.67 & 15.47 & 14.86 & 1.44 & $\mathrm{~W}$ \\
\hline SDSS J052522.85+000325.8 & 81.3452 & 0.0572 & 19.37 & 17.97 & 16.47 & 15.60 & 1.80 & $\mathrm{~W}$ \\
\hline SDSS J052526.69+003256.9 & 81.3612 & 0.5492 & 17.42 & 16.03 & 14.68 & 13.95 & 1.31 & $\mathrm{~W}$ \\
\hline SDSS J052532.56+005813.0 & 81.3857 & 0.9703 & 17.01 & 15.59 & 14.45 & 13.80 & 0.90 & $\mathrm{~W}$ \\
\hline SDSS J052538.97+005957.1 & 81.4124 & 0.9992 & 18.82 & 17.38 & 16.43 & 15.91 & 0.88 & $\mathrm{~W}$ \\
\hline SDSS J052540.77+010930.4 & 81.4199 & 1.1585 & 17.80 & 16.39 & 15.08 & 14.30 & 0.73 & $\mathrm{~W}$ \\
\hline SDSS J052542.40+005345.2 & 81.4267 & 0.8959 & 18.50 & 17.11 & 15.75 & 15.00 & 0.98 & $\mathrm{~W}$ \\
\hline SDSS J052600.63+004951.3 & 81.5026 & 0.8309 & 18.42 & 17.01 & 16.14 & 15.75 & 1.07 & $\mathrm{~W}$ \\
\hline SDSS J052600.74-004216.1 & 81.5031 & -0.7045 & 19.34 & 17.93 & 16.38 & 15.51 & 2.57 & $\mathrm{~W}$ \\
\hline SDSS J052602.29+004528.8 & 81.5096 & 0.7580 & 19.37 & 17.92 & 16.51 & 15.83 & 1.14 & $\mathrm{~W}$ \\
\hline SDSS J052605.29-004227.5 & 81.5221 & -0.7077 & 17.57 & 16.19 & 15.15 & 14.55 & 2.58 & $\mathrm{~W}$ \\
\hline SDSS J052605.45+011017.1 & 81.5228 & 1.1714 & 16.85 & 15.53 & 14.73 & 14.28 & 0.76 & $\mathrm{~W}$ \\
\hline SDSS J052607.74+002624.2 & 81.5323 & 0.4401 & 19.08 & 17.67 & 16.10 & 15.25 & 1.45 & $\mathrm{~W}$ \\
\hline SDSS J052610.25-001847.5 & 81.5427 & -0.3132 & 21.05 & 19.55 & 17.69 & 16.70 & 2.19 & $\mathrm{C}$ \\
\hline SDSS J052610.30-002247.9 & 81.5429 & -0.3800 & 17.82 & 16.43 & 15.35 & 14.72 & 2.26 & $\mathrm{~W}$ \\
\hline SDSS J052611.22+005425.9 & 81.5468 & 0.9072 & 18.87 & 17.45 & 16.33 & 15.72 & 1.01 & $\mathrm{~W}$ \\
\hline SDSS J052612.03+005223.9 & 81.5501 & 0.8733 & 20.50 & 18.95 & 17.19 & 16.24 & 1.04 & $\mathrm{~W}$ \\
\hline SDSS J052613.73-004347.7 & 81.5572 & -0.7299 & 19.13 & 17.63 & 16.75 & 16.25 & 2.61 & $\mathrm{~W}$ \\
\hline SDSS J052618.12+005114.8 & 81.5755 & 0.8541 & 20.99 & 19.43 & 17.74 & 16.91 & 1.07 & $\mathrm{C}$ \\
\hline SDSS J052625.73+005835.8 & 81.6072 & 0.9766 & 17.29 & 15.84 & 14.60 & 13.88 & 0.97 & $\mathrm{~W}$ \\
\hline
\end{tabular}


Table 2-Continued

\begin{tabular}{|c|c|c|c|c|c|c|c|c|}
\hline Name & RA & DEC & $g$ & $r$ & $i$ & $z$ & $\mathrm{R}^{\mathrm{b}}$ & Class $^{\mathrm{C}}$ \\
\hline SDSS J052636.70+000424.8 & 81.6529 & 0.0736 & 17.89 & 16.53 & 15.05 & 14.28 & 1.84 & $\mathrm{~W}$ \\
\hline SDSS J052642.28+003553.7 & 81.6762 & 0.5983 & 18.89 & 17.51 & 16.14 & 15.37 & 1.34 & $\mathrm{~W}$ \\
\hline SDSS J052642.86+000527.7 & 81.6786 & 0.0910 & 18.49 & 17.13 & 15.65 & 14.82 & 1.83 & $\mathrm{~W}$ \\
\hline SDSS J052643.92+000526.5 & 81.6830 & 0.0907 & 19.28 & 17.82 & 16.36 & 15.61 & 1.83 & $\mathrm{~W}$ \\
\hline SDSS J052649.55+005654.6 & 81.7065 & 0.9485 & 20.02 & 18.56 & 16.80 & 15.89 & 1.04 & $\mathrm{~W}$ \\
\hline SDSS J052649.69+010056.2 & 81.7071 & 1.0156 & 17.83 & 16.42 & 15.05 & 14.32 & 0.98 & $\mathrm{~W}$ \\
\hline SDSS J052700.12+010136.8 & 81.7505 & 1.0269 & 18.07 & 16.82 & 15.52 & 14.72 & 1.00 & $\mathrm{~W}$ \\
\hline SDSS J052704.34+000339.9 & 81.7681 & 0.0611 & 17.50 & 16.11 & 14.74 & 13.97 & 1.88 & W \\
\hline SDSS J052705.67+000317.2 & 81.7736 & 0.0548 & 17.53 & 16.15 & 14.86 & 14.09 & 1.89 & $\mathrm{~W}$ \\
\hline SDSS J052708.59+001051.7 & 81.7858 & 0.1810 & 20.09 & 18.68 & 16.86 & 15.93 & 1.77 & $\mathrm{~W}$ \\
\hline SDSS J052719.49-003928.1 & 81.8312 & -0.6578 & 16.89 & 15.49 & 14.60 & 14.08 & 2.59 & $\mathrm{~W}$ \\
\hline SDSS J052720.93+004623.1 & 81.8372 & 0.7731 & 19.15 & 17.71 & 16.22 & 15.41 & 1.26 & $\mathrm{~W}$ \\
\hline SDSS J052725.93+005107.7 & 81.8581 & 0.8521 & 17.32 & 15.89 & 14.89 & 14.26 & 1.20 & $\mathrm{C}$ \\
\hline SDSS J052728.87+003720.9 & 81.8703 & 0.6225 & 18.81 & 17.37 & 15.84 & 15.05 & 1.40 & $\mathrm{~W}$ \\
\hline SDSS J052730.70-010716.8 & 81.8779 & -1.1214 & 19.84 & 18.33 & 17.17 & 16.52 & 3.05 & $\mathrm{~W}$ \\
\hline SDSS J052738.25+002303.9 & 81.9094 & 0.3844 & 16.57 & 15.17 & 14.25 & 13.71 & 1.63 & $\mathrm{~W}$ \\
\hline SDSS J052749.35+011444.6 & 81.9557 & 1.2457 & 19.15 & 17.75 & 16.32 & 15.51 & 0.98 & W \\
\hline SDSS J052749.90+000712.4 & 81.9579 & 0.1201 & 17.65 & 16.26 & 15.02 & 14.41 & 1.89 & $\mathrm{~W}$ \\
\hline SDSS J052753.54+010831.9 & 81.9731 & 1.1422 & 18.37 & 17.00 & 15.51 & 14.66 & 1.06 & $\mathrm{~W}$ \\
\hline SDSS J052754.71+010718.0 & 81.9780 & 1.1217 & 19.24 & 17.85 & 16.51 & 15.77 & 1.07 & W \\
\hline SDSS J052756.04+001459.5 & 81.9835 & 0.2499 & 19.21 & 17.87 & 16.26 & 15.44 & 1.79 & W \\
\hline SDSS J052804.23+001237.7 & 82.0177 & 0.2105 & 17.82 & 16.45 & 15.16 & 14.42 & 1.84 & $\mathrm{~W}$ \\
\hline SDSS J052805.53+005404.9 & 82.0231 & 0.9014 & 18.21 & 16.81 & 15.54 & 14.80 & 1.26 & $\mathrm{~W}$ \\
\hline SDSS J052806.91-001806.0 & 82.0288 & -0.3017 & 18.47 & 17.06 & 15.74 & 14.98 & 2.31 & W \\
\hline SDSS J052808.56-011227.3 & 82.0357 & -1.2076 & 16.72 & 15.34 & 14.59 & 14.21 & 3.17 & $\mathrm{~W}$ \\
\hline SDSS J052810.18-004101.5 & 82.0424 & -0.6838 & 19.47 & 17.98 & 16.41 & 15.45 & 2.67 & W \\
\hline SDSS J052810.32-000333.0 & 82.0430 & -0.0592 & 19.18 & 17.80 & 16.18 & 15.26 & 2.09 & $\mathrm{~W}$ \\
\hline SDSS J052810.50+000530.7 & 82.0438 & 0.0919 & 18.27 & 16.88 & 15.63 & 14.99 & 1.95 & $\mathrm{~W}$ \\
\hline SDSS J052815.12-010259.3 & 82.0630 & -1.0498 & 18.66 & 17.24 & 15.72 & 14.94 & 3.03 & $\mathrm{~W}$ \\
\hline SDSS J052826.91-002505.2 & 82.1121 & -0.4181 & 18.56 & 17.12 & 15.79 & 15.01 & 2.45 & $\mathrm{~W}$ \\
\hline SDSS J052841.84-003556.8 & 82.1743 & -0.5991 & 17.17 & 15.79 & 14.80 & 14.28 & 2.64 & $\mathrm{~W}$ \\
\hline SDSS J052843.04-000008.4 & 82.1794 & -0.0023 & 18.67 & 17.35 & 16.14 & 15.56 & 2.10 & $\mathrm{~W}$ \\
\hline SDSS J052902.29-002606.1 & 82.2596 & -0.4350 & 19.60 & 18.12 & 16.54 & 15.66 & 2.52 & W \\
\hline SDSS J052906.11-004127.4 & 82.2755 & -0.6909 & 17.28 & 15.88 & 14.95 & 14.41 & 2.76 & $\mathrm{~W}$ \\
\hline SDSS J052907.90-003105.6 & 82.2829 & -0.5182 & 18.68 & 17.07 & 16.23 & 15.81 & 2.61 & W \\
\hline SDSS J052912.90+011340.1 & 82.3038 & 1.2278 & 19.62 & 18.25 & 16.76 & 15.98 & 1.28 & $\mathrm{C}$ \\
\hline SDSS J052930.65-005039.5 & 82.3777 & -0.8443 & 17.53 & 16.13 & 15.27 & 14.75 & 2.94 & $\mathrm{~W}$ \\
\hline SDSS J052939.46-003801.4 & 82.4144 & -0.6337 & 19.68 & 18.26 & 16.57 & 15.58 & 2.77 & $\mathrm{C}$ \\
\hline SDSS J052952.33-002023.3 & 82.4681 & -0.3398 & 20.41 & 18.99 & 17.15 & 16.04 & 2.54 & $\mathrm{~W}$ \\
\hline SDSS J052957.38+003923.6 & 82.4891 & 0.6566 & 16.71 & 15.34 & 14.47 & 13.97 & 1.76 & $\mathrm{~W}$ \\
\hline SDSS J052957.46-003951.8 & 82.4894 & -0.6644 & 17.69 & 16.29 & 15.23 & 14.62 & 2.83 & $\mathrm{~W}$ \\
\hline SDSS J052958.23+004612.6 & 82.4926 & 0.7702 & 18.69 & 17.23 & 15.94 & 15.31 & 1.69 & $\mathrm{~W}$ \\
\hline SDSS J052959.50+002728.6 & 82.4979 & 0.4580 & 17.92 & 16.50 & 15.31 & 14.65 & 1.91 & $\mathrm{~W}$ \\
\hline SDSS J053010.18-002801.6 & 82.5424 & -0.4671 & 20.95 & 19.48 & 17.63 & 16.71 & 2.68 & $\mathrm{C}$ \\
\hline SDSS J053010.48+001646.5 & 82.5437 & 0.2796 & 21.14 & 19.56 & 17.62 & 16.59 & 2.07 & $\mathrm{C}$ \\
\hline SDSS J053012.90-003841.4 & 82.5538 & -0.6449 & 18.89 & 17.39 & 15.89 & 14.99 & 2.84 & $\mathrm{~W}$ \\
\hline
\end{tabular}


Table 2-Continued

\begin{tabular}{|c|c|c|c|c|c|c|c|c|}
\hline Name & $\mathrm{RA}$ & DEC & $g$ & $r$ & $i$ & $z$ & $\mathrm{R}^{\mathrm{b}}$ & Class $^{\mathrm{C}}$ \\
\hline SDSS J053013.39-010049.1 & 82.5558 & -1.0136 & 19.30 & 17.91 & 15.98 & 14.92 & 3.17 & $\mathrm{~W}$ \\
\hline SDSS J053013.77+004519.6 & 82.5574 & 0.7554 & 18.85 & 17.45 & 16.57 & 16.18 & 1.75 & $\mathrm{~W}$ \\
\hline SDSS J053019.68-004959.7 & 82.5820 & -0.8333 & 20.42 & 18.70 & 17.02 & 15.98 & 3.02 & $\mathrm{C}$ \\
\hline SDSS J053026.54+010057.8 & 82.6106 & 1.0161 & 18.15 & 16.89 & 15.47 & 14.65 & 1.65 & $\mathrm{C}$ \\
\hline SDSS J053027.18-011431.1 & 82.6133 & -1.2420 & 18.33 & 16.94 & 15.66 & 14.96 & 3.40 & $\mathrm{~W}$ \\
\hline SDSS J053028.68-002052.2 & 82.6195 & -0.3478 & 19.47 & 18.08 & 16.65 & 15.86 & 2.62 & $\mathrm{C}$ \\
\hline SDSS J053034.20-001237.8 & 82.6425 & -0.2105 & 17.86 & 16.43 & 15.21 & 14.53 & 2.52 & W \\
\hline SDSS J053043.48+005200.5 & 82.6812 & 0.8668 & 19.17 & 17.75 & 16.83 & 16.36 & 1.79 & $\mathrm{~W}$ \\
\hline SDSS J053043.60-005236.7 & 82.6817 & -0.8769 & 20.34 & 18.97 & 17.15 & 16.16 & 3.11 & $\mathrm{~W}$ \\
\hline SDSS J053045.44+000905.4 & 82.6894 & 0.1515 & 19.87 & 18.44 & 17.01 & 16.23 & 2.27 & W \\
\hline SDSS J053047.46-010941.3 & 82.6978 & -1.1615 & 18.53 & 17.18 & 15.79 & 15.00 & 3.37 & W \\
\hline SDSS J053056.02+004138.3 & 82.7334 & 0.6940 & 17.89 & 16.47 & 15.12 & 14.40 & 1.93 & $\mathrm{~W}$ \\
\hline SDSS J053104.30+005617.7 & 82.7679 & 0.9383 & 19.03 & 17.51 & 16.46 & 16.02 & 1.82 & W \\
\hline SDSS J053107.98-003318.5 & 82.7833 & -0.5552 & 19.83 & 18.23 & 16.81 & 15.80 & 2.89 & $\mathrm{~W}$ \\
\hline SDSS J053114.19-002230.1 & 82.8092 & -0.3750 & 21.07 & 19.47 & 17.48 & 16.33 & 2.75 & $\mathrm{~W}$ \\
\hline SDSS J053116.57+001239.6 & 82.8191 & 0.2110 & 18.38 & 16.92 & 16.05 & 15.64 & 2.31 & $\mathrm{~W}$ \\
\hline SDSS J053121.96+000341.0 & 82.8415 & 0.0614 & 18.26 & 16.87 & 15.38 & 14.58 & 2.43 & $\mathrm{~W}$ \\
\hline SDSS J053124.25-010043.9 & 82.8511 & -1.0122 & 17.24 & 15.85 & 14.70 & 14.01 & 3.31 & $\mathrm{~W}$ \\
\hline SDSS J053135.48+004447.2 & 82.8979 & 0.7465 & 18.49 & 17.00 & 15.45 & 14.63 & 2.03 & $\mathrm{~W}$ \\
\hline SDSS J053147.19+001205.9 & 82.9466 & 0.2016 & 18.66 & 17.17 & 15.69 & 14.86 & 2.41 & $\mathrm{~W}$ \\
\hline SDSS J053147.88-003536.7 & 82.9495 & -0.5935 & 20.87 & 19.50 & 17.68 & 16.51 & 3.01 & $\mathrm{~W}$ \\
\hline SDSS J053206.53+005443.4 & 83.0272 & 0.9121 & 19.43 & 18.13 & 16.96 & 16.38 & 2.06 & $\mathrm{~W}$ \\
\hline SDSS J053207.81+001704.9 & 83.0326 & 0.2847 & 19.97 & 18.08 & 16.56 & 15.66 & 2.42 & $\mathrm{C}$ \\
\hline SDSS J053214.90-001102.9 & 83.0621 & -0.1842 & 19.18 & 17.76 & 15.93 & 14.91 & 2.76 & $\mathrm{~W}$ \\
\hline SDSS J053215.50-002637.6 & 83.0646 & -0.4438 & 19.03 & 17.65 & 15.92 & 15.07 & 2.96 & W \\
\hline SDSS J053217.88-003141.5 & 83.0745 & -0.5282 & 18.71 & 17.00 & 15.87 & 15.12 & 3.03 & $\mathrm{C}$ \\
\hline SDSS J053217.95-003107.9 & 83.0748 & -0.5189 & 16.99 & 15.60 & 14.80 & 14.40 & 3.03 & $\mathrm{~W}$ \\
\hline SDSS J053218.47+003148.2 & 83.0770 & 0.5301 & 19.94 & 18.50 & 16.79 & 15.81 & 2.30 & $\mathrm{~W}$ \\
\hline SDSS J053219.25-010714.8 & 83.0802 & -1.1208 & 17.72 & 16.28 & 15.10 & 14.41 & 3.52 & $\mathrm{~W}$ \\
\hline SDSS J053219.62-003148.7 & 83.0818 & -0.5302 & 18.14 & 16.58 & 15.45 & 14.78 & 3.04 & $\mathrm{~W}$ \\
\hline SDSS J053221.81-000947.5 & 83.0909 & -0.1632 & 19.59 & 18.05 & 16.59 & 15.80 & 2.77 & $\mathrm{~W}$ \\
\hline SDSS J053223.88-002843.5 & 83.0995 & -0.4788 & 16.51 & 15.09 & 14.26 & 13.80 & 3.01 & $\mathrm{~W}$ \\
\hline SDSS J053224.32-010138.3 & 83.1014 & -1.0273 & 20.50 & 18.99 & 17.32 & 16.39 & 3.45 & $\mathrm{C}$ \\
\hline SDSS J053225.38-000626.1 & 83.1058 & -0.1073 & 19.96 & 18.54 & 17.20 & 16.49 & 2.74 & W \\
\hline SDSS J053228.79-010305.1 & 83.1200 & -1.0514 & 16.17 & 14.75 & 13.74 & 13.25 & 3.48 & $\mathrm{~W}$ \\
\hline SDSS J053229.38-010722.7 & 83.1224 & -1.1230 & 18.83 & 17.46 & 15.67 & 14.77 & 3.55 & $\mathrm{C}$ \\
\hline SDSS J053232.31-011418.9 & 83.1347 & -1.2386 & 19.21 & 17.75 & 16.17 & 15.36 & 3.65 & $\mathrm{~W}$ \\
\hline SDSS J053234.33-002701.9 & 83.1431 & -0.4505 & 18.61 & 17.21 & 15.56 & 14.66 & 3.02 & $\mathrm{~W}$ \\
\hline SDSS J053237.70-010932.7 & 83.1571 & -1.1591 & 20.86 & 19.35 & 17.69 & 16.80 & 3.60 & $\mathrm{~W}$ \\
\hline SDSS J053238.32-001941.4 & 83.1597 & -0.3282 & 19.24 & 17.85 & 16.18 & 15.22 & 2.94 & $\mathrm{~W}$ \\
\hline SDSS J053238.88-003317.0 & 83.1620 & -0.5547 & 17.20 & 15.79 & 14.74 & 14.13 & 3.11 & $\mathrm{~W}$ \\
\hline SDSS J053239.82-002356.3 & 83.1659 & -0.3990 & 17.61 & 16.19 & 14.94 & 14.22 & 2.99 & $\mathrm{~W}$ \\
\hline SDSS J053241.53-000923.7 & 83.1731 & -0.1566 & 20.51 & 19.01 & 17.17 & 16.13 & 2.82 & $\mathrm{C}$ \\
\hline SDSS J053249.91-002533.1 & 83.2080 & -0.4259 & 17.60 & 15.76 & 14.88 & 14.39 & 3.04 & $\mathrm{~W}$ \\
\hline SDSS J053250.45-003542.2 & 83.2102 & -0.5951 & 18.01 & 16.70 & 15.32 & 14.42 & 3.17 & $\mathrm{C}$ \\
\hline SDSS J053300.32-010531.9 & 83.2514 & -1.0922 & 17.64 & 16.24 & 15.06 & 14.41 & 3.59 & $\mathrm{~W}$ \\
\hline
\end{tabular}


Table 2-Continued

\begin{tabular}{|c|c|c|c|c|c|c|c|c|}
\hline Name & RA & DEC & $g$ & $r$ & $i$ & $z$ & $\mathrm{R}^{\mathrm{b}}$ & Class $^{\mathrm{C}}$ \\
\hline SDSS J053304.21+000327.0 & 83.2676 & 0.0575 & 19.37 & 17.91 & 16.25 & 15.34 & 2.74 & $\mathrm{C}$ \\
\hline SDSS J053306.33-001555.0 & 83.2764 & -0.2653 & 17.54 & 16.12 & 14.81 & 14.05 & 2.97 & $\mathrm{~W}$ \\
\hline SDSS J053306.56-002254.7 & 83.2773 & -0.3819 & 16.90 & 15.55 & 14.36 & 13.57 & 3.06 & $\mathrm{C}$ \\
\hline SDSS J053309.15-011028.5 & 83.2881 & -1.1746 & 18.23 & 16.80 & 15.56 & 14.84 & 3.68 & W \\
\hline SDSS J053311.20+001358.5 & 83.2967 & 0.2329 & 17.87 & 16.31 & 15.04 & 14.43 & 2.66 & $\mathrm{~W}$ \\
\hline SDSS J053311.30-003740.9 & 83.2971 & -0.6280 & 18.83 & 17.43 & 15.92 & 14.83 & 3.25 & $\mathrm{~W}$ \\
\hline SDSS J053317.77-004100.1 & 83.3241 & -0.6834 & 19.34 & 17.96 & 16.47 & 15.53 & 3.31 & $\mathrm{C}$ \\
\hline SDSS J053323.47+003730.6 & 83.3478 & 0.6252 & 19.18 & 17.57 & 16.34 & 15.74 & 2.48 & $\mathrm{~W}$ \\
\hline SDSS J053325.14-005637.0 & 83.3548 & -0.9436 & 18.51 & 17.15 & 15.76 & 14.97 & 3.53 & $\mathrm{~W}$ \\
\hline SDSS J053326.22-004044.3 & 83.3593 & -0.6790 & 19.39 & 18.00 & 16.23 & 15.22 & 3.33 & $\mathrm{~W}$ \\
\hline SDSS J053329.70-011041.9 & 83.3738 & -1.1783 & 16.73 & 15.35 & 14.59 & 14.18 & 3.73 & $\mathrm{~W}$ \\
\hline SDSS J053335.14-005535.1 & 83.3964 & -0.9264 & 19.63 & 18.02 & 16.52 & 15.60 & 3.55 & $\mathrm{C}$ \\
\hline SDSS J053337.74-005914.3 & 83.4073 & -0.9873 & 18.30 & 16.88 & 15.65 & 14.90 & 3.60 & $\mathrm{~W}$ \\
\hline SDSS J053341.27-002953.7 & 83.4220 & -0.4983 & 17.05 & 15.64 & 14.48 & 13.84 & 3.24 & $\mathrm{~W}$ \\
\hline SDSS J053342.46-000739.0 & 83.4270 & -0.1275 & 18.43 & 17.06 & 15.72 & 15.01 & 2.99 & $\mathrm{~W}$ \\
\hline SDSS J053344.74-004340.4 & 83.4364 & -0.7279 & 19.62 & 18.23 & 16.82 & 16.02 & 3.42 & $\mathrm{~W}$ \\
\hline SDSS J053347.46+003602.4 & 83.4478 & 0.6007 & 19.27 & 17.84 & 16.08 & 15.06 & 2.58 & $\mathrm{~W}$ \\
\hline SDSS J053348.95-003921.2 & 83.4540 & -0.6559 & 18.86 & 17.38 & 16.50 & 15.98 & 3.38 & $\mathrm{~W}$ \\
\hline SDSS J053349.04-004625.9 & 83.4544 & -0.7739 & 16.81 & 15.44 & 14.29 & 13.63 & 3.47 & $\mathrm{~W}$ \\
\hline SDSS J053349.05-005520.2 & 83.4544 & -0.9223 & 16.66 & 15.28 & 14.36 & 13.84 & 3.58 & $\mathrm{~W}$ \\
\hline SDSS J053350.20-004239.6 & 83.4592 & -0.7110 & 19.35 & 17.94 & 16.17 & 15.05 & 3.42 & $\mathrm{C}$ \\
\hline SDSS J053351.78+000028.3 & 83.4657 & 0.0079 & 17.35 & 15.97 & 15.01 & 14.49 & 2.93 & $\mathrm{~W}$ \\
\hline SDSS J053400.81+000213.3 & 83.5034 & 0.0370 & 19.12 & 17.61 & 16.12 & 15.16 & 2.94 & $\mathrm{C}$ \\
\hline SDSS J053404.67+010228.5 & 83.5195 & 1.0413 & 18.68 & 17.37 & 16.46 & 16.09 & 2.47 & $\mathrm{~W}$ \\
\hline SDSS J053407.28-001626.5 & 83.5304 & -0.2740 & 19.30 & 17.88 & 16.16 & 15.22 & 3.16 & $\mathrm{~W}$ \\
\hline SDSS J053413.50-010720.8 & 83.5563 & -1.1224 & 17.61 & 16.19 & 15.10 & 14.46 & 3.80 & $\mathrm{~W}$ \\
\hline SDSS J053414.22-004243.8 & 83.5593 & -0.7122 & 20.55 & 19.04 & 17.21 & 16.09 & 3.49 & $\mathrm{C}$ \\
\hline SDSS J053415.29-011519.6 & 83.5637 & -1.2554 & 17.63 & 16.19 & 15.02 & 14.35 & 3.91 & $\mathrm{~W}$ \\
\hline SDSS J053426.37-003351.3 & 83.6099 & -0.5643 & 18.07 & 16.68 & 15.23 & 14.37 & 3.42 & $\mathrm{~W}$ \\
\hline SDSS J053427.01-005422.7 & 83.6126 & -0.9063 & 19.75 & 18.29 & 16.70 & 15.81 & 3.67 & $\mathrm{~W}$ \\
\hline SDSS J053427.15-001242.6 & 83.6132 & -0.2119 & 17.17 & 15.71 & 14.18 & 13.33 & 3.18 & $\mathrm{~W}$ \\
\hline SDSS J053431.08-011112.1 & 83.6295 & -1.1867 & 18.80 & 17.38 & 15.97 & 15.14 & 3.90 & $\mathrm{C}$ \\
\hline SDSS J053435.44+000340.4 & 83.6477 & 0.0612 & 19.59 & 18.05 & 16.50 & 15.56 & 3.04 & $\mathrm{~W}$ \\
\hline SDSS J053441.16+002647.0 & 83.6715 & 0.4464 & 16.63 & 15.26 & 14.26 & 13.69 & 2.85 & $\mathrm{~W}$ \\
\hline SDSS J053446.86-002612.6 & 83.6953 & -0.4368 & 16.48 & 15.09 & 14.14 & 13.57 & 3.39 & $\mathrm{~W}$ \\
\hline SDSS J053454.23+000415.4 & 83.7260 & 0.0710 & 17.81 & 16.38 & 15.41 & 14.86 & 3.10 & $\mathrm{~W}$ \\
\hline SDSS J053458.20-001502.4 & 83.7425 & -0.2507 & 19.24 & 17.83 & 16.16 & 15.24 & 3.31 & $\mathrm{~W}$ \\
\hline SDSS J053459.45-002717.6 & 83.7477 & -0.4549 & 20.10 & 18.71 & 17.09 & 16.31 & 3.44 & $\mathrm{C}$ \\
\hline SDSS J053501.77-005954.4 & 83.7574 & -0.9985 & 17.19 & 15.80 & 14.81 & 14.18 & 3.83 & $\mathrm{~W}$ \\
\hline SDSS J053503.57-001511.7 & 83.7649 & -0.2533 & 19.58 & 18.16 & 16.39 & 15.22 & 3.32 & $\mathrm{~W}$ \\
\hline SDSS J053506.59-002108.3 & 83.7775 & -0.3523 & 20.04 & 18.54 & 16.72 & 15.67 & 3.40 & $\mathrm{~W}$ \\
\hline SDSS J053515.61-003442.2 & 83.8151 & -0.5784 & 20.89 & 19.31 & 17.47 & 16.31 & 3.58 & $\mathrm{~W}$ \\
\hline SDSS J053518.00-001742.1 & 83.8250 & -0.2950 & 19.56 & 18.19 & 16.39 & 15.41 & 3.40 & $\mathrm{~W}$ \\
\hline SDSS J053525.22-004324.3 & 83.8551 & -0.7234 & 18.21 & 16.52 & 15.26 & 14.43 & 3.70 & $\mathrm{~W}$ \\
\hline SDSS J053527.88+001025.0 & 83.8662 & 0.1736 & 18.81 & 17.40 & 16.11 & 15.42 & 3.16 & $\mathrm{~W}$ \\
\hline SDSS J053532.90+000214.2 & 83.8871 & 0.0373 & 20.41 & 19.00 & 17.40 & 16.54 & 3.25 & $\mathrm{~W}$ \\
\hline
\end{tabular}


Table 2-Continued

\begin{tabular}{|c|c|c|c|c|c|c|c|c|}
\hline Name & RA & DEC & $g$ & $r$ & $i$ & $z$ & $\mathrm{R}^{\mathrm{b}}$ & Class $^{\mathrm{C}}$ \\
\hline SDSS J053532.98-003114.1 & 83.8874 & -0.5206 & 17.78 & 16.37 & 15.13 & 14.46 & 3.59 & $\mathrm{~W}$ \\
\hline SDSS J053536.66-000459.4 & 83.9028 & -0.0832 & 20.59 & 19.16 & 17.38 & 16.32 & 3.33 & $\mathrm{~W}$ \\
\hline SDSS J053537.51-010624.0 & 83.9063 & -1.1067 & 19.06 & 17.62 & 16.23 & 15.47 & 4.02 & W \\
\hline SDSS J053538.51-005111.5 & 83.9105 & -0.8532 & 19.38 & 17.67 & 16.06 & 15.27 & 3.84 & $\mathrm{C}$ \\
\hline SDSS J053543.97-005609.7 & 83.9332 & -0.9361 & 17.53 & 16.13 & 14.89 & 14.13 & 3.91 & $\mathrm{~W}$ \\
\hline SDSS J053544.61-001426.4 & 83.9359 & -0.2407 & 16.78 & 15.39 & 14.46 & 13.94 & 3.45 & $\mathrm{~W}$ \\
\hline SDSS J053551.70-000956.0 & 83.9654 & -0.1656 & 18.90 & 17.38 & 15.76 & 14.81 & 3.43 & $\mathrm{~W}$ \\
\hline SDSS J053555.54+003339.0 & 83.9814 & 0.5608 & 20.56 & 19.06 & 17.36 & 16.41 & 3.07 & $\mathrm{C}$ \\
\hline SDSS J053557.13-003933.6 & 83.9881 & -0.6594 & 19.30 & 17.93 & 16.44 & 15.61 & 3.76 & W \\
\hline SDSS J053600.82+010716.7 & 84.0034 & 1.1213 & 18.85 & 17.34 & 16.43 & 15.96 & 2.91 & $\mathrm{~W}$ \\
\hline SDSS J053609.19-002632.1 & 84.0383 & -0.4422 & 18.14 & 16.74 & 15.29 & 14.48 & 3.66 & $\mathrm{~W}$ \\
\hline SDSS J053610.40-004039.1 & 84.0433 & -0.6775 & 19.53 & 18.12 & 16.63 & 15.83 & 3.81 & $\mathrm{~W}$ \\
\hline SDSS J053618.37+003644.6 & 84.0766 & 0.6124 & 19.17 & 17.83 & 16.42 & 15.64 & 3.14 & $\mathrm{~W}$ \\
\hline SDSS J053623.67+000041.6 & 84.0986 & 0.0116 & 20.11 & 18.71 & 17.28 & 16.58 & 3.44 & $\mathrm{~W}$ \\
\hline SDSS J053642.89-004508.3 & 84.1787 & -0.7523 & 18.79 & 17.40 & 15.81 & 14.91 & 3.96 & $\mathrm{~W}$ \\
\hline SDSS J053650.26+003654.8 & 84.2094 & 0.6152 & 19.56 & 18.09 & 16.84 & 16.18 & 3.26 & $\mathrm{~W}$ \\
\hline SDSS J053650.72+000742.3 & 84.2114 & 0.1284 & 17.85 & 16.45 & 15.21 & 14.54 & 3.48 & $\mathrm{~W}$ \\
\hline SDSS J053655.09-003022.9 & 84.2296 & -0.5064 & 17.89 & 16.50 & 15.24 & 14.55 & 3.85 & W \\
\hline SDSS J053701.00-005512.3 & 84.2542 & -0.9201 & 19.02 & 17.68 & 16.23 & 15.44 & 4.13 & $\mathrm{~W}$ \\
\hline SDSS J053704.36+005516.2 & 84.2682 & 0.9212 & 18.27 & 17.30 & 15.55 & 14.66 & 3.22 & $\mathrm{~W}$ \\
\hline SDSS J053704.83-005814.1 & 84.2702 & -0.9706 & 19.12 & 17.65 & 16.26 & 15.46 & 4.18 & $\mathrm{~W}$ \\
\hline SDSS J053704.97-000812.4 & 84.2707 & -0.1368 & 19.89 & 18.45 & 16.74 & 15.87 & 3.67 & $\mathrm{~W}$ \\
\hline SDSS J053709.92-011050.4 & 84.2913 & -1.1807 & 18.74 & 17.37 & 15.77 & 14.92 & 4.34 & $\mathrm{~W}$ \\
\hline SDSS J053710.85+010423.1 & 84.2952 & 1.0731 & 19.70 & 18.25 & 16.79 & 15.96 & 3.20 & $\mathrm{~W}$ \\
\hline SDSS J053716.27-010336.5 & 84.3178 & -1.0602 & 21.26 & 19.84 & 17.76 & 16.70 & 4.27 & $\mathrm{C}$ \\
\hline SDSS J053719.30+010555.0 & 84.3304 & 1.0986 & 19.21 & 17.77 & 16.20 & 15.24 & 3.23 & $\mathrm{~W}$ \\
\hline SDSS J053723.38-010344.3 & 84.3474 & -1.0623 & 16.57 & 15.18 & 14.22 & 13.67 & 4.30 & $\mathrm{~W}$ \\
\hline SDSS J053724.24-002350.4 & 84.3510 & -0.3973 & 19.19 & 17.79 & 16.48 & 15.75 & 3.88 & $\mathrm{~W}$ \\
\hline SDSS J053725.62-003206.4 & 84.3568 & -0.5351 & 19.54 & 17.96 & 16.64 & 15.77 & 3.96 & $\mathrm{C}$ \\
\hline SDSS J053729.79+000540.9 & 84.3741 & 0.0947 & 17.65 & 16.13 & 15.22 & 14.75 & 3.64 & $\mathrm{~W}$ \\
\hline SDSS J053729.80-002414.4 & 84.3742 & -0.4040 & 17.26 & 15.86 & 14.53 & 13.78 & 3.90 & $\mathrm{~W}$ \\
\hline SDSS J053729.83+010124.6 & 84.3743 & 1.0235 & 21.16 & 19.84 & 17.94 & 16.74 & 3.29 & $\mathrm{C}$ \\
\hline SDSS J053730.46+004104.0 & 84.3769 & 0.6845 & 17.75 & 16.34 & 15.11 & 14.39 & 3.39 & $\mathrm{~W}$ \\
\hline SDSS J053731.91-001916.8 & 84.3830 & -0.3214 & 19.38 & 17.47 & 16.02 & 15.22 & 3.86 & $\mathrm{C}$ \\
\hline SDSS J053735.75-002640.3 & 84.3990 & -0.4445 & 20.43 & 19.04 & 17.33 & 16.48 & 3.94 & $\mathrm{~W}$ \\
\hline SDSS J053737.35-002827.8 & 84.4057 & -0.4744 & 19.61 & 18.17 & 16.28 & 15.26 & 3.97 & $\mathrm{~W}$ \\
\hline SDSS J053738.97-004656.4 & 84.4124 & -0.7823 & 19.48 & 18.03 & 16.37 & 15.46 & 4.16 & $\mathrm{~W}$ \\
\hline SDSS J053742.93+002434.5 & 84.4289 & 0.4096 & 17.46 & 16.05 & 15.12 & 14.55 & 3.54 & $\mathrm{~W}$ \\
\hline SDSS J053747.50+011540.9 & 84.4479 & 1.2614 & 17.07 & 15.72 & 14.88 & 14.39 & 3.31 & $\mathrm{~W}$ \\
\hline SDSS J053747.92-000603.9 & 84.4497 & -0.1011 & 19.50 & 18.09 & 16.53 & 15.71 & 3.80 & $\mathrm{~W}$ \\
\hline SDSS J053749.15+000923.6 & 84.4548 & 0.1566 & 19.59 & 18.18 & 16.62 & 15.77 & 3.68 & $\mathrm{~W}$ \\
\hline SDSS J053750.64+004322.3 & 84.4610 & 0.7229 & 19.68 & 18.30 & 16.80 & 16.00 & 3.46 & $\mathrm{~W}$ \\
\hline SDSS J053752.16+005827.1 & 84.4674 & 0.9742 & 19.26 & 16.78 & 15.53 & 14.85 & 3.39 & $\mathrm{~W}$ \\
\hline SDSS J053758.23+003155.3 & 84.4926 & 0.5320 & 17.33 & 15.94 & 14.76 & 14.07 & 3.56 & $\mathrm{~W}$ \\
\hline SDSS J053801.02-010858.3 & 84.5043 & -1.1496 & 19.63 & 18.06 & 16.47 & 15.57 & 4.47 & $\mathrm{~W}$ \\
\hline SDSS J053801.50+000701.5 & 84.5063 & 0.1171 & 18.17 & 16.77 & 15.50 & 14.78 & 3.74 & $\mathrm{~W}$ \\
\hline
\end{tabular}


Table 2-Continued

\begin{tabular}{|c|c|c|c|c|c|c|c|c|}
\hline Name & RA & DEC & $g$ & $r$ & $i$ & $z$ & $\mathrm{R}^{\mathrm{b}}$ & Class $^{\mathrm{c}}$ \\
\hline SDSS J053806.65+002251.1 & 84.5277 & 0.3809 & 17.79 & 16.39 & 15.19 & 14.52 & 3.65 & $\mathrm{~W}$ \\
\hline SDSS J053806.74-010817.8 & 84.5281 & -1.1383 & 20.41 & 19.01 & 17.23 & 16.10 & 4.48 & $\mathrm{~W}$ \\
\hline SDSS J053808.51-001156.3 & 84.5355 & -0.1990 & 19.94 & 18.57 & 16.74 & 15.77 & 3.92 & $\mathrm{~W}$ \\
\hline SDSS J053812.51-000908.8 & 84.5522 & -0.1525 & 17.04 & 15.61 & 14.66 & 14.08 & 3.91 & $\mathrm{~W}$ \\
\hline SDSS J053812.72-001228.1 & 84.5530 & -0.2078 & 17.55 & 16.14 & 14.93 & 14.23 & 3.94 & $\mathrm{C}$ \\
\hline SDSS J053817.30-010010.5 & 84.5721 & -1.0029 & 18.90 & 17.49 & 15.72 & 14.68 & 4.42 & $\mathrm{C}$ \\
\hline SDSS J053821.31-001830.4 & 84.5888 & -0.3085 & 20.10 & 18.67 & 16.89 & 15.88 & 4.03 & $\mathrm{~W}$ \\
\hline SDSS J053829.54-004104.8 & 84.6231 & -0.6847 & 17.95 & 16.56 & 15.11 & 14.21 & 4.27 & $\mathrm{~W}$ \\
\hline SDSS J053835.53-001221.5 & 84.6481 & -0.2060 & 20.47 & 18.95 & 17.21 & 16.06 & 4.02 & $\mathrm{C}$ \\
\hline SDSS J053837.34+001948.9 & 84.6556 & 0.3303 & 19.34 & 17.84 & 16.65 & 15.93 & 3.78 & $\mathrm{C}$ \\
\hline SDSS J053839.62+001407.9 & 84.6651 & 0.2356 & 20.59 & 19.14 & 17.31 & 16.34 & 3.83 & W \\
\hline SDSS J053841.68-000234.4 & 84.6737 & -0.0429 & 18.17 & 16.82 & 15.61 & 14.90 & 3.96 & $\mathrm{~W}$ \\
\hline SDSS J053846.10-004620.5 & 84.6921 & -0.7724 & 19.75 & 18.34 & 16.37 & 15.20 & 4.38 & $\mathrm{~W}$ \\
\hline SDSS J053848.20-000051.0 & 84.7009 & -0.0142 & 17.74 & 16.38 & 15.19 & 14.46 & 3.98 & $\mathrm{~W}$ \\
\hline SDSS J053851.37-002429.4 & 84.7141 & -0.4082 & 19.87 & 18.43 & 16.94 & 16.09 & 4.19 & $\mathrm{~W}$ \\
\hline SDSS J053853.84-004920.0 & 84.7244 & -0.8222 & 17.51 & 16.14 & 14.64 & 13.56 & 4.43 & $\mathrm{~W}$ \\
\hline SDSS J053904.29-003810.0 & 84.7679 & -0.6361 & 18.19 & 16.76 & 15.34 & 14.48 & 4.36 & $\mathrm{~W}$ \\
\hline SDSS J053908.87+003045.3 & 84.7870 & 0.5126 & 18.85 & 17.48 & 15.79 & 14.91 & 3.84 & $\mathrm{~W}$ \\
\hline SDSS J053909.18-002353.3 & 84.7883 & -0.3982 & 17.52 & 16.17 & 15.06 & 14.37 & 4.24 & $\mathrm{~W}$ \\
\hline SDSS J053914.59-011124.4 & 84.8108 & -1.1901 & 17.53 & 16.15 & 15.24 & 14.75 & 4.73 & $\mathrm{~W}$ \\
\hline SDSS J053914.96+010959.5 & 84.8124 & 1.1665 & 18.13 & 16.66 & 15.48 & 14.75 & 3.69 & $\mathrm{~W}$ \\
\hline SDSS J053918.17+011132.8 & 84.8257 & 1.1925 & 18.90 & 17.39 & 16.51 & 16.02 & 3.69 & $\mathrm{~W}$ \\
\hline SDSS J053918.74-001305.3 & 84.8281 & -0.2182 & 18.77 & 17.35 & 16.04 & 15.23 & 4.18 & $\mathrm{~W}$ \\
\hline SDSS J053919.91+010138.6 & 84.8330 & 1.0274 & 18.82 & 16.97 & 16.14 & 15.70 & 3.73 & $\mathrm{C}$ \\
\hline SDSS J053925.88+011334.6 & 84.8578 & 1.2263 & 19.09 & 17.64 & 16.56 & 15.92 & 3.72 & $\mathrm{~W}$ \\
\hline SDSS J053938.75+010947.1 & 84.9115 & 1.1631 & 19.77 & 18.32 & 16.72 & 15.84 & 3.78 & $\mathrm{~W}$ \\
\hline SDSS J053940.26-003149.9 & 84.9178 & -0.5305 & 19.42 & 17.68 & 16.58 & 15.91 & 4.42 & $\mathrm{C}$ \\
\hline SDSS J053941.02-001716.8 & 84.9210 & -0.2880 & 18.59 & 17.15 & 15.82 & 15.08 & 4.30 & $\mathrm{C}$ \\
\hline SDSS J053941.76-011435.6 & 84.9240 & -1.2432 & 19.31 & 17.84 & 16.28 & 15.39 & 4.85 & $\mathrm{~W}$ \\
\hline SDSS J053950.20+001748.2 & 84.9592 & 0.2967 & 19.19 & 17.78 & 16.34 & 15.53 & 4.08 & $\mathrm{~W}$ \\
\hline SDSS J053952.65+001406.4 & 84.9694 & 0.2351 & 20.30 & 18.55 & 17.08 & 16.33 & 4.11 & $\mathrm{~W}$ \\
\hline SDSS J054010.45+001729.7 & 85.0436 & 0.2916 & 19.77 & 18.33 & 16.84 & 16.10 & 4.16 & $\mathrm{~W}$ \\
\hline SDSS J054021.92-001906.9 & 85.0913 & -0.3186 & 18.44 & 17.03 & 15.76 & 15.06 & 4.46 & $\mathrm{~W}$ \\
\hline SDSS J054048.80+010056.2 & 85.2034 & 1.0156 & 18.42 & 17.41 & 15.93 & 15.30 & 4.10 & $\mathrm{~W}$ \\
\hline SDSS J054058.03+003329.0 & 85.2418 & 0.5581 & 17.05 & 15.66 & 14.79 & 14.28 & 4.25 & $\mathrm{~W}$ \\
\hline SDSS J054059.22+011107.4 & 85.2468 & 1.1854 & 20.70 & 19.23 & 17.52 & 16.59 & 4.11 & $\mathrm{~W}$ \\
\hline SDSS J054103.34-004213.5 & 85.2639 & -0.7038 & 16.91 & 15.52 & 14.24 & 13.47 & 4.81 & $\mathrm{~W}$ \\
\hline SDSS J054105.34+011336.8 & 85.2723 & 1.2269 & 18.13 & 16.80 & 15.48 & 14.81 & 4.13 & $\mathrm{~W}$ \\
\hline SDSS J054107.73+001453.6 & 85.2822 & 0.2482 & 19.18 & 17.79 & 16.24 & 15.54 & 4.39 & $\mathrm{C}$ \\
\hline SDSS J054113.59+003930.1 & 85.3066 & 0.6584 & 19.34 & 17.67 & 16.75 & 16.30 & 4.29 & $\mathrm{~W}$ \\
\hline SDSS J054118.28-002213.9 & 85.3262 & -0.3705 & 19.48 & 18.02 & 16.64 & 15.88 & 4.69 & $\mathrm{~W}$ \\
\hline SDSS J054119.72+001849.2 & 85.3322 & 0.3137 & 18.73 & 17.31 & 16.12 & 15.45 & 4.42 & $\mathrm{~W}$ \\
\hline SDSS J054123.09-000854.3 & 85.3462 & -0.1484 & 18.51 & 17.10 & 15.77 & 15.05 & 4.61 & $\mathrm{~W}$ \\
\hline SDSS J054123.86-010408.7 & 85.3495 & -1.0691 & 18.76 & 17.42 & 15.81 & 14.99 & 5.08 & $\mathrm{~W}$ \\
\hline SDSS J054128.86+003951.7 & 85.3703 & 0.6644 & 17.73 & 16.05 & 15.09 & 14.51 & 4.35 & $\mathrm{~W}$ \\
\hline SDSS J054144.34-003519.1 & 85.4348 & -0.5887 & 18.95 & 17.43 & 15.97 & 15.05 & 4.90 & $\mathrm{~W}$ \\
\hline
\end{tabular}


Table 2-Continued

\begin{tabular}{|c|c|c|c|c|c|c|c|c|}
\hline Name & RA & $\mathrm{DEC}$ & $g$ & $r$ & $i$ & $z$ & $\mathrm{R}^{\mathrm{b}}$ & Class $^{\mathrm{c}}$ \\
\hline SDSS J054145.35+001256.3 & 85.4390 & 0.2157 & 16.91 & 15.62 & 14.96 & 14.65 & 4.55 & $\mathrm{~W}$ \\
\hline SDSS J054200.37-004528.5 & 85.5016 & -0.7579 & 21.10 & 19.69 & 17.53 & 16.17 & 5.04 & $\mathrm{~W}$ \\
\hline SDSS J054204.19+010908.9 & 85.5175 & 1.1525 & 20.00 & 18.40 & 17.31 & 16.70 & 4.38 & $\mathrm{~W}$ \\
\hline SDSS J054220.47-010424.9 & 85.5853 & -1.0736 & 18.17 & 16.66 & 15.69 & 15.21 & 5.28 & $\mathrm{~W}$ \\
\hline SDSS J054227.25-010924.4 & 85.6136 & -1.1568 & 19.12 & 17.64 & 16.26 & 15.50 & 5.35 & $\mathrm{~W}$ \\
\hline SDSS J054227.45-010315.9 & 85.6144 & -1.0544 & 17.51 & 15.73 & 14.81 & 14.30 & 5.29 & $\mathrm{~W}$ \\
\hline SDSS J054228.10-010349.3 & 85.6171 & -1.0637 & 18.26 & 16.80 & 16.02 & 15.60 & 5.30 & $\mathrm{~W}$ \\
\hline SDSS J054228.64-000044.4 & 85.6193 & -0.0123 & 19.16 & 17.61 & 16.60 & 15.99 & 4.80 & $\mathrm{~W}$ \\
\hline SDSS J054229.09-010321.5 & 85.6212 & -1.0560 & 15.88 & 14.59 & 13.89 & 13.56 & 5.30 & $\mathrm{~W}$ \\
\hline SDSS J054230.98-000126.9 & 85.6291 & -0.0241 & 20.24 & 18.59 & 16.56 & 15.44 & 4.82 & $\mathrm{~W}$ \\
\hline SDSS J054233.34-002550.4 & 85.6389 & -0.4307 & 18.06 & 16.70 & 15.31 & 14.50 & 5.00 & $\mathrm{~W}$ \\
\hline SDSS J054238.76-011025.6 & 85.6615 & -1.1738 & 19.26 & 17.25 & 16.19 & 15.50 & 5.40 & $\mathrm{~W}$ \\
\hline SDSS J054244.57-010446.8 & 85.6857 & -1.0797 & 17.69 & 15.40 & 14.25 & 13.46 & 5.37 & $\mathrm{~W}$ \\
\hline SDSS J054251.34+010206.8 & 85.7139 & 1.0352 & 18.97 & 17.49 & 16.14 & 15.21 & 4.60 & $\mathrm{C}$ \\
\hline SDSS J054254.19-010506.3 & 85.7258 & -1.0851 & 20.58 & 18.17 & 16.64 & 15.56 & 5.40 & $\mathrm{~W}$ \\
\hline SDSS J054303.64+000631.3 & 85.7652 & 0.1087 & 17.13 & 15.74 & 15.13 & 14.88 & 4.90 & $\mathrm{~W}$ \\
\hline SDSS J054305.68+001453.6 & 85.7737 & 0.2482 & 16.75 & 15.36 & 14.38 & 13.83 & 4.86 & $\mathrm{C}$ \\
\hline SDSS J054306.32+005223.8 & 85.7764 & 0.8733 & 18.26 & 16.76 & 15.32 & 14.46 & 4.69 & $\mathrm{~W}$ \\
\hline SDSS J054309.71+001202.2 & 85.7905 & 0.2006 & 20.62 & 19.12 & 17.37 & 16.43 & 4.89 & $\mathrm{~W}$ \\
\hline SDSS J054311.76-003539.8 & 85.7990 & -0.5944 & 20.47 & 18.91 & 17.43 & 16.48 & 5.22 & $\mathrm{~W}$ \\
\hline SDSS J054319.67+000528.6 & 85.8320 & 0.0913 & 18.89 & 17.33 & 16.53 & 16.09 & 4.96 & $\mathrm{~W}$ \\
\hline SDSS J054333.48-010057.9 & 85.8895 & -1.0161 & 19.12 & 17.24 & 16.12 & 15.46 & 5.50 & $\mathrm{C}$ \\
\hline SDSS J054344.49+010406.4 & 85.9354 & 1.0684 & 20.10 & 18.52 & 17.32 & 16.71 & 4.81 & $\mathrm{~W}$ \\
\hline SDSS J054348.41+001937.5 & 85.9518 & 0.3271 & 18.58 & 17.18 & 16.03 & 15.36 & 5.00 & $\mathrm{~W}$ \\
\hline SDSS J054350.07+004454.4 & 85.9586 & 0.7484 & 18.12 & 16.65 & 15.54 & 14.97 & 4.89 & $\mathrm{~W}$ \\
\hline SDSS J054351.87+003639.0 & 85.9661 & 0.6109 & 17.18 & 15.79 & 14.48 & 13.67 & 4.93 & $\mathrm{~W}$ \\
\hline SDSS J054358.43-004438.0 & 85.9935 & -0.7439 & 19.51 & 17.49 & 16.22 & 15.36 & 5.46 & $\mathrm{~W}$ \\
\hline SDSS J054424.87+010815.2 & 86.1037 & 1.1376 & 18.93 & 17.37 & 16.56 & 16.09 & 4.97 & $\mathrm{~W}$ \\
\hline SDSS J054428.06-010525.3 & 86.1169 & -1.0904 & 20.03 & 17.72 & 16.29 & 15.34 & 5.74 & $\mathrm{~W}$ \\
\hline SDSS J054435.59-010107.2 & 86.1483 & -1.0187 & 20.12 & 18.09 & 16.85 & 16.02 & 5.73 & $\mathrm{~W}$ \\
\hline SDSS J054437.55-001650.9 & 86.1565 & -0.2808 & 17.67 & 16.26 & 15.26 & 14.69 & 5.40 & $\mathrm{~W}$ \\
\hline SDSS J054450.07-003017.9 & 86.2086 & -0.5050 & 20.65 & 19.12 & 17.20 & 16.15 & 5.54 & $\mathrm{C}$ \\
\hline SDSS J054454.86-004055.1 & 86.2286 & -0.6820 & 19.53 & 17.57 & 16.60 & 15.97 & 5.64 & $\mathrm{~W}$ \\
\hline SDSS J054501.89+000630.9 & 86.2579 & 0.1086 & 19.93 & 18.33 & 16.49 & 15.39 & 5.36 & $\mathrm{~W}$ \\
\hline SDSS J054503.46+003454.6 & 86.2644 & 0.5818 & 20.45 & 18.93 & 17.11 & 16.12 & 5.23 & $\mathrm{~W}$ \\
\hline SDSS J054504.38+003712.0 & 86.2683 & 0.6200 & 19.58 & 18.14 & 16.38 & 15.45 & 5.23 & $\mathrm{~W}$ \\
\hline SDSS J054509.50+005418.3 & 86.2896 & 0.9051 & 20.51 & 19.02 & 17.11 & 16.03 & 5.19 & $\mathrm{~W}$ \\
\hline SDSS J054510.17-003512.3 & 86.2924 & -0.5868 & 19.22 & 17.66 & 16.09 & 14.98 & 5.65 & $\mathrm{C}$ \\
\hline SDSS J054526.16+000637.9 & 86.3590 & 0.1105 & 17.78 & 16.30 & 14.85 & 14.06 & 5.45 & $\mathrm{~W}$ \\
\hline SDSS J054538.90-004555.8 & 86.4121 & -0.7655 & 20.32 & 18.85 & 17.24 & 16.34 & 5.84 & $\mathrm{~W}$ \\
\hline SDSS J054544.64+001300.5 & 86.4360 & 0.2168 & 16.49 & 15.09 & 14.30 & 13.87 & 5.49 & $\mathrm{~W}$ \\
\hline SDSS J054557.93+000248.5 & 86.4914 & 0.0468 & 19.66 & 18.20 & 16.53 & 15.49 & 5.60 & $\mathrm{C}$ \\
\hline SDSS J054559.34+002920.7 & 86.4973 & 0.4891 & 16.50 & 14.99 & 13.70 & 13.06 & 5.48 & $\mathrm{~W}$ \\
\hline SDSS J054600.17+000307.1 & 86.5007 & 0.0520 & 18.34 & 16.67 & 14.88 & 13.87 & 5.61 & $\mathrm{~W}$ \\
\hline SDSS J054601.37-000013.6 & 86.5057 & -0.0038 & 19.29 & 17.27 & 16.02 & 15.24 & 5.63 & $\mathrm{C}$ \\
\hline SDSS J054604.92+000145.5 & 86.5205 & 0.0293 & 18.70 & 16.79 & 15.58 & 14.76 & 5.63 & $\mathrm{~W}$ \\
\hline
\end{tabular}


Table 2-Continued

\begin{tabular}{|c|c|c|c|c|c|c|c|c|}
\hline Name & RA & DEC & $g$ & $r$ & $i$ & $z$ & $\mathrm{R}^{\mathrm{b}}$ & Class $^{\mathrm{c}}$ \\
\hline SDSS J054606.02+003702.6 & 86.5251 & 0.6174 & 17.95 & 16.41 & 15.29 & 14.73 & 5.48 & $\mathrm{~W}$ \\
\hline SDSS J054608.66+000454.3 & 86.5361 & 0.0818 & 20.30 & 17.92 & 16.50 & 15.49 & 5.63 & $\mathrm{~W}$ \\
\hline SDSS J054609.26+001332.5 & 86.5386 & 0.2257 & 16.94 & 15.29 & 14.16 & 13.57 & 5.59 & $\mathrm{C}$ \\
\hline SDSS J054621.44+000454.7 & 86.5894 & 0.0819 & 17.15 & 15.64 & 14.87 & 14.62 & 5.68 & $\mathrm{~W}$ \\
\hline SDSS J054625.88+000931.8 & 86.6079 & 0.1589 & 19.54 & 17.82 & 16.25 & 15.21 & 5.68 & $\mathrm{C}$ \\
\hline SDSS J054626.65+003107.3 & 86.6111 & 0.5187 & 19.85 & 17.46 & 16.03 & 14.95 & 5.58 & $\mathrm{C}$ \\
\hline SDSS J054638.40+001511.5 & 86.6600 & 0.2532 & 21.64 & 19.47 & 17.09 & 15.69 & 5.70 & $\mathrm{C}$ \\
\hline SDSS J054638.57+002205.9 & 86.6607 & 0.3683 & 21.13 & 18.63 & 16.91 & 15.73 & 5.67 & $\mathrm{C}$ \\
\hline SDSS J054639.14-000914.9 & 86.6631 & -0.1541 & 18.02 & 15.58 & 14.07 & 13.15 & 5.83 & $\mathrm{~W}$ \\
\hline SDSS J054640.77+002722.7 & 86.6699 & 0.4563 & 20.35 & 18.48 & 16.58 & 15.17 & 5.65 & $\mathrm{C}$ \\
\hline SDSS J054645.31-002943.6 & 86.6888 & -0.4955 & 18.59 & 16.50 & 15.30 & 14.51 & 5.98 & $\mathrm{~W}$ \\
\hline SDSS J054650.16-000342.1 & 86.7090 & -0.0617 & 18.10 & 15.73 & 14.30 & 13.26 & 5.84 & $\mathrm{~W}$ \\
\hline SDSS J054651.33+003225.6 & 86.7139 & 0.5405 & 18.51 & 16.09 & 14.65 & 13.64 & 5.68 & $\mathrm{~W}$ \\
\hline SDSS J054652.92+005929.3 & 86.7205 & 0.9915 & 19.48 & 18.03 & 16.86 & 16.17 & 5.60 & $\mathrm{~W}$ \\
\hline SDSS J054656.54+002052.7 & 86.7356 & 0.3480 & 18.24 & 16.11 & 14.84 & 13.95 & 5.75 & $\mathrm{~W}$ \\
\hline SDSS J054706.48-005916.0 & 86.7770 & -0.9878 & 19.66 & 17.32 & 15.97 & 15.12 & 6.27 & $\mathrm{~W}$ \\
\hline SDSS J054706.98+003155.9 & 86.7791 & 0.5322 & 20.76 & 18.43 & 16.65 & 15.44 & 5.74 & $\mathrm{C}$ \\
\hline SDSS J054716.95+003719.0 & 86.8206 & 0.6220 & 20.62 & 18.00 & 16.40 & 15.23 & 5.76 & $\mathrm{~W}$ \\
\hline SDSS J054717.16+001824.5 & 86.8215 & 0.3068 & 19.87 & 17.91 & 16.40 & 15.37 & 5.84 & $\mathrm{C}$ \\
\hline SDSS J054719.18+001920.5 & 86.8299 & 0.3224 & 20.58 & 18.20 & 16.50 & 15.48 & 5.84 & $\mathrm{C}$ \\
\hline SDSS J054721.17-005736.7 & 86.8382 & -0.9602 & 19.55 & 16.80 & 15.21 & 14.16 & 6.31 & $\mathrm{~W}$ \\
\hline SDSS J054724.89-005350.9 & 86.8537 & -0.8975 & 19.88 & 18.32 & 17.05 & 16.39 & 6.29 & $\mathrm{~W}$ \\
\hline SDSS J054728.28-010002.0 & 86.8679 & -1.0006 & 20.20 & 17.80 & 16.54 & 15.70 & 6.35 & W \\
\hline SDSS J054733.78-003106.1 & 86.8908 & -0.5184 & 18.19 & 16.59 & 15.33 & 14.64 & 6.17 & $\mathrm{~W}$ \\
\hline SDSS J054743.22-010032.4 & 86.9301 & -1.0090 & 20.45 & 18.00 & 16.67 & 15.86 & 6.41 & $\mathrm{~W}$ \\
\hline SDSS J054744.99+003841.8 & 86.9375 & 0.6450 & 19.69 & 16.77 & 14.92 & 13.63 & 5.87 & $\mathrm{~W}$ \\
\hline SDSS J054747.30+002226.3 & 86.9471 & 0.3740 & 19.65 & 17.14 & 15.82 & 14.91 & 5.94 & $\mathrm{~W}$ \\
\hline SDSS J054748.05-010148.0 & 86.9502 & -1.0300 & 19.87 & 17.41 & 16.09 & 15.32 & 6.44 & $\mathrm{~W}$ \\
\hline SDSS J054748.63+004707.7 & 86.9527 & 0.7855 & 20.84 & 18.25 & 16.80 & 15.75 & 5.86 & $\mathrm{~W}$ \\
\hline SDSS J054750.11+004154.5 & 86.9588 & 0.6985 & 19.59 & 17.88 & 16.55 & 15.89 & 5.88 & $\mathrm{~W}$ \\
\hline SDSS J054750.34+004702.7 & 86.9598 & 0.7841 & 18.42 & 16.69 & 15.81 & 15.32 & 5.87 & $\mathrm{~W}$ \\
\hline SDSS J054753.30+010219.1 & 86.9721 & 1.0387 & 19.90 & 17.96 & 16.77 & 15.96 & 5.84 & $\mathrm{~W}$ \\
\hline SDSS J054753.66+004320.4 & 86.9736 & 0.7224 & 18.22 & 16.58 & 15.18 & 14.45 & 5.89 & $\mathrm{~W}$ \\
\hline SDSS J054754.35-005317.7 & 86.9765 & -0.8883 & 19.80 & 16.48 & 14.72 & 13.39 & 6.40 & $\mathrm{~W}$ \\
\hline SDSS J054754.42-003907.6 & 86.9768 & -0.6521 & 18.76 & 17.29 & 16.07 & 15.38 & 6.30 & $\mathrm{~W}$ \\
\hline SDSS J054754.83+001538.6 & 86.9785 & 0.2607 & 18.23 & 16.69 & 15.73 & 15.26 & 6.00 & $\mathrm{~W}$ \\
\hline SDSS J054755.78-005902.0 & 86.9824 & -0.9839 & 20.83 & 18.36 & 17.13 & 16.26 & 6.45 & $\mathrm{~W}$ \\
\hline SDSS J054758.01+001341.0 & 86.9917 & 0.2281 & 19.81 & 17.98 & 17.00 & 16.38 & 6.02 & $\mathrm{~W}$ \\
\hline SDSS J054802.14+004940.9 & 87.0089 & 0.8280 & 19.29 & 16.84 & 15.61 & 14.73 & 5.91 & $\mathrm{~W}$ \\
\hline SDSS J054807.22+004908.1 & 87.0301 & 0.8189 & 19.24 & 17.22 & 16.17 & 15.51 & 5.93 & $\mathrm{~W}$ \\
\hline SDSS J054808.67+002153.9 & 87.0362 & 0.3650 & 19.61 & 17.51 & 16.53 & 15.91 & 6.03 & $\mathrm{~W}$ \\
\hline SDSS J054809.64+002246.5 & 87.0402 & 0.3796 & 20.36 & 18.08 & 16.96 & 16.23 & 6.03 & $\mathrm{~W}$ \\
\hline SDSS J054811.78+004152.9 & 87.0491 & 0.6980 & 19.86 & 17.80 & 16.65 & 15.89 & 5.97 & $\mathrm{~W}$ \\
\hline SDSS J054814.57-005645.5 & 87.0607 & -0.9460 & 20.02 & 18.52 & 16.72 & 15.69 & 6.50 & $\mathrm{~W}$ \\
\hline SDSS J054817.78-003917.7 & 87.0741 & -0.6549 & 17.71 & 16.29 & 14.85 & 13.96 & 6.40 & $\mathrm{~W}$ \\
\hline SDSS J054842.62-002313.5 & 87.1776 & -0.3871 & 20.10 & 18.64 & 17.04 & 16.14 & 6.39 & $\mathrm{~W}$ \\
\hline
\end{tabular}


Table 2-Continued

\begin{tabular}{|c|c|c|c|c|c|c|c|c|}
\hline Name & RA & DEC & $g$ & $r$ & $i$ & $z$ & $\mathrm{R}^{\mathrm{b}}$ & Class $^{\mathrm{C}}$ \\
\hline SDSS J054851.07-003006.0 & 87.2128 & -0.5017 & 17.53 & 16.13 & 15.43 & 15.12 & 6.47 & $\mathrm{~W}$ \\
\hline SDSS J054909.75-010829.3 & 87.2907 & -1.1415 & 20.44 & 17.62 & 16.01 & 14.81 & 6.79 & $\mathrm{~W}$ \\
\hline SDSS J054914.57-000714.8 & 87.3107 & -0.1208 & 17.69 & 16.29 & 15.35 & 14.90 & 6.43 & $\mathrm{~W}$ \\
\hline SDSS J054943.60+005638.6 & 87.4317 & 0.9441 & 18.69 & 17.12 & 16.04 & 15.44 & 6.31 & $\mathrm{C}$ \\
\hline SDSS J054946.93+003102.8 & 87.4456 & 0.5175 & 18.09 & 15.74 & 14.31 & 13.40 & 6.40 & $\mathrm{~W}$ \\
\hline SDSS J054952.28-001147.9 & 87.4679 & -0.1966 & 18.81 & 17.35 & 16.14 & 15.51 & 6.60 & $\mathrm{~W}$ \\
\hline SDSS J054956.21-004843.7 & 87.4842 & -0.8122 & 19.12 & 17.71 & 15.96 & 15.00 & 6.83 & $\mathrm{~W}$ \\
\hline SDSS J054958.08+000417.7 & 87.4920 & 0.0716 & 21.26 & 19.36 & 17.48 & 16.33 & 6.55 & $\mathrm{C}$ \\
\hline SDSS J054959.02+005550.5 & 87.4959 & 0.9307 & 19.74 & 18.22 & 16.76 & 15.95 & 6.37 & $\mathrm{~W}$ \\
\hline SDSS J055004.32+005624.3 & 87.5180 & 0.9401 & 18.75 & 17.14 & 16.24 & 15.88 & 6.39 & $\mathrm{~W}$ \\
\hline SDSS J055005.55+002305.7 & 87.5232 & 0.3849 & 19.14 & 17.63 & 15.93 & 14.97 & 6.50 & $\mathrm{~W}$ \\
\hline SDSS J055010.21+010156.0 & 87.5426 & 1.0322 & 18.32 & 16.96 & 15.92 & 15.63 & 6.40 & $\mathrm{~W}$ \\
\hline SDSS J055010.38-000241.3 & 87.5433 & -0.0448 & 19.36 & 17.53 & 16.11 & 15.26 & 6.63 & $\mathrm{~W}$ \\
\hline SDSS J055020.01+001032.6 & 87.5834 & 0.1757 & 20.72 & 19.06 & 17.03 & 15.84 & 6.61 & $\mathrm{~W}$ \\
\hline SDSS J055038.14+002232.0 & 87.6590 & 0.3756 & 20.45 & 18.96 & 17.34 & 16.44 & 6.63 & $\mathrm{~W}$ \\
\hline SDSS J055041.06-011201.0 & 87.6711 & -1.2003 & 18.69 & 17.33 & 16.21 & 15.71 & 7.16 & $\mathrm{~W}$ \\
\hline SDSS J055115.65+003911.4 & 87.8152 & 0.6532 & 18.07 & 16.58 & 15.23 & 14.49 & 6.73 & W \\
\hline SDSS J055124.33-010512.6 & 87.8514 & -1.0869 & 18.12 & 16.65 & 15.62 & 15.10 & 7.28 & $\mathrm{~W}$ \\
\hline SDSS J055124.86-005109.2 & 87.8536 & -0.8526 & 18.53 & 16.81 & 15.88 & 15.32 & 7.19 & $\mathrm{~W}$ \\
\hline SDSS J055133.61-010651.4 & 87.8901 & -1.1143 & 18.09 & 16.60 & 15.54 & 14.99 & 7.33 & $\mathrm{~W}$ \\
\hline SDSS J055135.15+000120.5 & 87.8965 & 0.0224 & 19.33 & 17.82 & 16.60 & 15.97 & 6.95 & $\mathrm{~W}$ \\
\hline SDSS J055135.72-010322.2 & 87.8989 & -1.0562 & 15.86 & 14.18 & 13.41 & 13.01 & 7.31 & $\mathrm{~W}$ \\
\hline SDSS J055211.45+011119.8 & 88.0477 & 1.1889 & 19.07 & 17.37 & 16.35 & 15.68 & 6.89 & $\mathrm{~W}$ \\
\hline SDSS J055237.20+002431.9 & 88.1550 & 0.4089 & 17.81 & 16.34 & 15.20 & 14.54 & 7.11 & W \\
\hline SDSS J055238.73-002614.8 & 88.1614 & -0.4375 & 18.29 & 16.80 & 15.62 & 15.00 & 7.34 & $\mathrm{~W}$ \\
\hline SDSS J055244.28-002526.5 & 88.1845 & -0.4240 & 19.29 & 17.69 & 16.67 & 16.11 & 7.35 & W \\
\hline SDSS J055255.95+002125.6 & 88.2331 & 0.3571 & 18.69 & 17.26 & 15.91 & 15.16 & 7.20 & $\mathrm{~W}$ \\
\hline SDSS J055341.87-004041.1 & 88.4245 & -0.6781 & 20.66 & 18.78 & 17.55 & 16.79 & 7.66 & $\mathrm{~W}$ \\
\hline SDSS J055401.49-004411.9 & 88.5062 & -0.7367 & 18.38 & 16.93 & 15.75 & 15.13 & 7.76 & $\mathrm{~W}$ \\
\hline SDSS J055452.48+011138.3 & 88.7187 & 1.1940 & 19.45 & 17.98 & 16.63 & 15.96 & 7.56 & $\mathrm{C}$ \\
\hline SDSS J055505.24-004508.8 & 88.7718 & -0.7524 & 19.41 & 17.95 & 16.67 & 15.98 & 8.02 & $\mathrm{~W}$ \\
\hline SDSS J055512.26+002408.3 & 88.8011 & 0.4023 & 19.47 & 18.09 & 16.74 & 16.01 & 7.75 & $\mathrm{~W}$ \\
\hline SDSS J055552.60-001945.9 & 88.9692 & -0.3294 & 17.64 & 16.22 & 15.01 & 14.34 & 8.08 & W \\
\hline SDSS J055623.10-004419.7 & 89.0963 & -0.7388 & 19.88 & 17.91 & 16.80 & 16.12 & 8.32 & $\mathrm{~W}$ \\
\hline SDSS J055629.02+004835.3 & 89.1209 & 0.8098 & 19.54 & 18.09 & 16.48 & 15.62 & 8.00 & W \\
\hline SDSS J055631.89-002110.1 & 89.1329 & -0.3528 & 20.23 & 18.63 & 17.26 & 16.51 & 8.24 & $\mathrm{~W}$ \\
\hline SDSS J055734.48-001356.4 & 89.3937 & -0.2324 & 20.39 & 18.86 & 17.38 & 16.54 & 8.46 & $\mathrm{~W}$ \\
\hline SDSS J055742.29-005017.2 & 89.4262 & -0.8381 & 19.98 & 18.57 & 17.26 & 16.59 & 8.66 & $\mathrm{~W}$ \\
\hline SDSS J055742.93-010234.1 & 89.4289 & -1.0428 & 19.13 & 17.58 & 16.65 & 16.17 & 8.73 & W \\
\hline SDSS J055801.82-000303.8 & 89.5076 & -0.0511 & 20.53 & 19.06 & 17.26 & 16.20 & 8.53 & $\mathrm{~W}$ \\
\hline SDSS J055806.59-004946.0 & 89.5275 & -0.8295 & 19.09 & 17.65 & 16.10 & 15.29 & 8.76 & $\mathrm{~W}$ \\
\hline SDSS J055823.09+000405.9 & 89.5962 & 0.0683 & 17.23 & 15.77 & 14.31 & 13.50 & 8.59 & $\mathrm{~W}$ \\
\hline SDSS J055835.30-004729.1 & 89.6471 & -0.7914 & 20.73 & 19.13 & 17.75 & 16.97 & 8.86 & $\mathrm{C}$ \\
\hline SDSS J055854.84+005636.6 & 89.7285 & 0.9435 & 19.20 & 17.75 & 16.26 & 15.47 & 8.59 & $\mathrm{~W}$ \\
\hline SDSS J055912.55-002947.3 & 89.8023 & -0.4965 & 19.42 & 17.68 & 16.72 & 16.13 & 8.93 & $\mathrm{~W}$ \\
\hline SDSS J055938.44+004035.7 & 89.9102 & 0.6766 & 17.25 & 15.81 & 15.04 & 14.73 & 8.80 & W \\
\hline
\end{tabular}


Table 2-Continued

\begin{tabular}{lllllllll}
\hline \hline Name & $\mathrm{RA}$ & $\mathrm{DEC}$ & $g$ & $r$ & $i$ & $z$ & $\mathrm{R}^{\mathrm{b}}$ & Class $^{\mathrm{c}}$ \\
\hline
\end{tabular}

${ }^{a}$ Distance in degrees from the Be star 25 Orionis.

${ }^{\mathrm{b}}$ Preliminary classification as WTTS (W) or CTTS (C) based on multi-band variability. 